\title{
Receptor-interacting protein kinases modulate noise-induced sensory hair cell death
}

\author{
H-W Zheng ${ }^{1,2}$, J Chen ${ }^{1}$ and S-H Sha ${ }^{\star, 1}$
}

Receptor-interacting protein (RIP) kinases promote the induction of necrotic cell death pathways. Here we investigated signaling pathways in outer hair cells (OHCs) of adult male CBA/J mice exposed to noise that causes permanent threshold shifts, with a particular focus on RIP kinase-regulated necroptosis. One hour after noise exposure, nuclei of OHCs in the basal region of the cochlea displayed both apoptotic and necrotic features. RIP1 and RIP3 protein levels increased and caspase-8 was activated. Treatment with pan-caspase inhibitor ZVAD blocked the activation of caspase-8 and reduced the number of apoptotic nuclei, while increasing levels of RIP1, RIP3, and necrotic OHCs. Conversely, treatment with necrosis inhibitor necrostatin-1 (Nec-1) or RIP3 siRNA (siRIP3) diminished noise-induced increases in RIP1 and RIP3, and decreased necrotic OHC nuclei. This treatment also increased the number of apoptotic nuclei without increasing activation of caspase-8. Consistent with the elevation of levels of RIP1 and RIP3, noise-induced active AMPK $\alpha$ levels increased with ZVAD treatment, but decreased with Nec-1 and siRIP3 treatment. Furthermore, treatment with SiRIP3 did not alter the activation of caspase-8, but instead increased activation of caspase-9 and promoted endonuclease G translocation into OHC nuclei. Finally, auditory brainstem response functional measurements and morphological assessment of OHCs showed that ZVAD treatment reduces noise-induced deficits. This protective function is potentiated when combined with siRIP3 treatment. In conclusion, noise-induced OHC apoptosis and necrosis are modulated by caspases and RIP kinases, respectively. Inhibition of either pathway shifts the prevalence of $\mathrm{OHC}$ death to the alternative pathway.

Cell Death and Disease (2014) 5, e1262; doi:10.1038/cddis.2014.177; published online 29 May 2014

Subject Category: Neuroscience

The receptor-interacting protein 1 (RIP1) and RIP3 kinases can mediate the initiation of necrotic cell death pathways via physical and functional interactions. ${ }^{1-4}$ RIP kinase family members are essential sensors of cellular stress that share a homologous $\mathrm{N}$-terminal kinase domain but differ in their recruitment domains. The death domain of RIP1 binds to death receptors, such as tumor necrosis factor (TNF) receptor 1 (TNFR-1), to form a signaling complex with caspase-8. ${ }^{5}$ In response to death receptor stimulation, caspase- 8 is activated through a cleavage step, and subsequently inactivates RIP1. ${ }^{6}$ This process leads to apoptosis and can be prevented by caspase inhibition. ${ }^{7}$ Alternatively, RIP1 can be activated directly by interacting with RIP3 through the RIP homotypic interaction motif. This leads to cellular ATP depletion and necrotic cell death. ${ }^{8-11}$

Energy depletion has long been implicated in the pathogenesis of noise-induced hearing loss (NIHL), which is mediated primarily by the demise of outer hair cells (OHCs) in the cochlea. Decreased ATP levels within cochlear tissues have been detected in NIHL. ${ }^{12,13}$ Likewise, the ATP content of the organ of Corti decreases under ischemic conditions. ${ }^{14}$ In response to ATP depletion, AMP-activated protein kinase (AMPK), a crucial cellular energy sensor, is activated via phosphorylation on the threonine 172 (T172) residue of its catalytic $\alpha$-subunit, which has been suggested to result in necrotic $\mathrm{OHC}$ death. ${ }^{15}$

In contrast, ATP-consuming processes are rapidly shut off by caspases in apoptosis, a process that has been well documented in noise-induced $\mathrm{OHC}$ death. ${ }^{16}$ This mode of cell death results from a bio-energetic and metabolic catastrophe coupled to multiple active execution mechanisms. In classical extrinsic apoptotic signaling pathways, active caspase- 8 is generated at the receptosome complex, resulting in direct activation of downstream pro-caspases. In this pathway, a serial process leads to caspase- 9 activation. ${ }^{17}$ Alternatively, endonuclease $G$ (EndoG) can translocate to the nucleus, where it is capable of mediating large-scale DNA fragmentation independent of caspase activity. ${ }^{18,19}$ The activation of caspase-8, caspase-9, and EndoG translocation have all been documented in noise-induced $\mathrm{OHC}$ death. ${ }^{20-24}$

\footnotetext{
${ }^{1}$ Department of Pathology and Laboratory Medicine, Medical University of South Carolina, Charleston, SC 29425, USA

${ }^{*}$ Corresponding author: S-H Sha, Department of Pathology and Laboratory Medicine, Medical University of South Carolina, Walton Research Building, Room 403-E, 39 Sabin Street, Charleston, SC 29425, USA. Tel: +1 843792 8324; Fax: +1 843792 0368; E-mail: shasu@musc.edu

${ }^{2}$ Permanent address: Department of Otolaryngology-Head and Neck Surgery, Beijing University People's Hospital, 11 Xizhimen South Street, Xicheng District, Beijing 100853, China

Keywords: RIP-kinases; necrotic or apoptotic hair cell death; noise-induced hearing loss; interaction of apoptotic and necrotic hair cell death

Abbreviations: ABR, auditory brainstem response; AMPK, AMP-activated protein kinase; BBN, broadband noise; CC8, cleaved caspase-8; CC9, cleaved caspase-9; EndoG, endonuclease G; IP, intraperitoneal; Nec-1, necrostatin-1; NIHL, noise-induced hearing loss; OHC, outer hair cell; PI, propidium iodide; RIP, receptor-interacting protein; siControl, scrambled-siRNA control; siRIP3, RIP3 siRNA; TNF, tumor necrosis factor; TNFR-1, tumor necrosis factor receptor 1; ZVAD, pan-caspase inhibitor ZVAD
} 
In this study, we investigated both apoptotic and necrotic $\mathrm{OHC}$ death pathways, and their potential interactions in adult male CBA/J mice exposed to traumatic noise, with a particular focus on RIP kinases. Pharmacological cell death inhibitors pan-caspase inhibitor ZVAD and necrosis inhibitor necrostatin-1 (Nec-1) and RIP3 silencing techniques were used to assess the modulation of $\mathrm{OHC}$ death. Finally, the effectiveness of ZVAD and RIP3 siRNA (siRIP3), individually and in combination, in attenuating NIHL was evaluated. Our results provide insights into the molecular mechanisms modulating noise-induced necrosis and apoptosis in OHCs and identify potential therapeutic targets for prevention of NIHL.

\section{Results}

Noise induced both apoptotic and necrotic changes in nuclei of OHCs. We have previously shown that exposure of CBA $/ \mathrm{J}$ mice to broadband noise (BBN) at $106 \mathrm{~dB}$ SPL resulted in permanent hearing loss and a base-to-apex gradient of sensory hair cell loss after 2 weeks, without damage to supporting cells and spiral ganglion cells. Under this noise condition, both apoptosis and necrosis of OHCs were evident in the lower basal turn of the cochlea $1 \mathrm{~h}$ after noise exposure, but not in the upper basal, middle, and apical turns. ${ }^{15}$ Here we expanded on our previous observation and evaluated the extent of loss by conducting a more detailed assessment of morphological criteria in conjunction with propidium iodide $(\mathrm{PI})$ uptake in the cochlear epithelia. No condensed or swollen OHC nuclei were observed in control mice that were not exposed to noise. One hour after noise exposure, some OHCs in the lower basal turn $(4.5-5.5 \mathrm{~mm}$ from apex) of the cochleae displayed condensed apoptotic nuclei (Figure 1a, panel: Noise, arrows) or swollen necrotic nuclei (Figure 1a, panel: Noise, arrowheads). Quantification of noise-induced condensed, swollen, and missing OHCs as the percentage of abnormal OHCs in the lower basal turn region of the cochlear epithelium revealed a significant difference (Figure 1b, $F_{2,15}=18.590, P<0.001$ ). Although the number of condensed $\mathrm{OHC}$ nuclei $(40.4 \% \pm 4.1)$ was not significantly different from swollen nuclei $(34.3 \% \pm 2.7$; $P=0.065)$, both were significantly greater than the number of missing nuclei $(25.3 \% \pm 2.8) 1 \mathrm{~h}$ after noise exposure (swollen versus missing: $P=0.007$; condensed versus missing: $P<0.001)$. There were no missing, apoptotic, or necrotic OHCs in control mice that were not exposed to noise.

A pharmacological apoptosis inhibitor decreased noise-induced apoptotic OHC nuclei and increased necrotic nuclei, while a necrosis inhibitor had the opposite effect. The pharmacological regulators ZVAD, an irreversible inhibitor of the caspase family of proteases, and necrosis inhibitor $\mathrm{Nec}-1$ were applied in an attempt to inhibit $\mathrm{OHC}$ death following noise exposure. First, the concentration of ZVAD and Nec-1 for local delivery to the mouse middle ear immediately after $106 \mathrm{~dB}$ SPL noise exposure was estimated by dose-toxicity studies in mouse organ of Corti explant cultures. The highest drug concentrations without damage to OHCs in culture (ZVAD: $100 \mathrm{mM}$ or
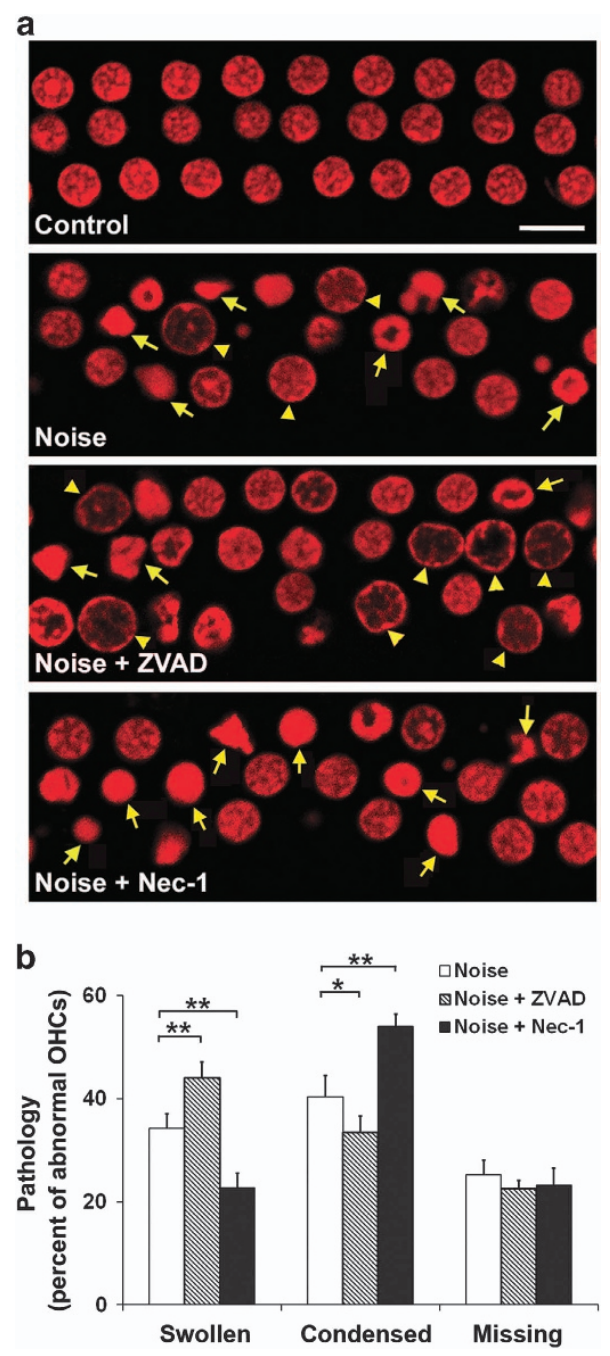

Figure 1 Noise-induced apoptotic and necrotic $\mathrm{OHC}$ death is modulated by inhibitors of apoptosis (ZVAD) and necrosis (Nec-1). (a) Representative images of $\mathrm{OHC}$ nuclei in the lower basal region of the cochlear epithelium. Control: normal $\mathrm{OHC}$ nuclei without prior exposure to noise. Noise: $\mathrm{OHCs}$ display both swollen and condensed nuclei following exposure to $106 \mathrm{~dB}$ SPL BBN. Noise + ZVAD: an increase in the number of swollen OHC nuclei is observed in noise-exposed mice treated with ZVAD by local delivery. Noise + Nec-1: an increase in condensed $\mathrm{OHC}$ nuclei appears in noise-exposed mice treated with $\mathrm{Nec}-1$ by local delivery. Red: PI labeling of OHC nuclei; arrowheads: swollen necrotic nuclei; arrows: condensed apoptotic nuclei. All images are representative of five individual ears for each condition. Scale bar $=10 \mu \mathrm{m}$. (b) Quantification of swollen or condensed $\mathrm{OHC}$ nuclei and missing OHCs in the lower basal turn of cochlear epithelium $1 \mathrm{~h}$ after noise exposure confirms a significant increase in the number of swollen nuclei and a decline in the number of condensed nuclei with local delivery of ZVAD, while administration of Nec-1 via local delivery causes the reverse effect. Data are presented as means + S.D.; $n=5$ for each condition; ${ }^{*} P<0.05$, ${ }^{* \star} P<0.001$

Nec-1: $300 \mu \mathrm{M})$ were then used in vivo. With ZVAD treatment, the percentage of noise-induced condensed $\mathrm{OHC}$ nuclei was significantly decreased in the lower basal turn (from $40.4 \%$ to $33.5 \%$ ) $1 \mathrm{~h}$ after exposure (Figure $1 \mathrm{~b}$, $t_{10}=3.288, P=0.008$ ), while the percentage of swollen nuclei significantly increased (from $34.3 \%$ to $44 \%$ ) (Figure $1 \mathrm{~b}, t_{10}=-5.675, P<0.001$ ). In contrast, Nec-1 treatment resulted in the opposite trend, a significant 
increase in noise-induced condensed nuclei (from $40.4 \%$ to $54.4 \%$ ) (Figure $1 b, t_{10}=-7.138, P<0.001$ ) and a decrease in swollen nuclei (from $34.3 \%$ to $22.7 \%$ ) (Figure $1 \mathrm{~b}$, $\left.t_{10}=7.305, P<0.001\right)$. The percentage of missing $\mathrm{OHCs}$ induced by noise did not change after treatment with either inhibitor $\left(F_{2,12}=2.539, P=0.12\right)$ (Figure $\left.1 b\right)$.

Noise-induced changes in RIP levels in OHCs were modulated by apoptosis and necrosis inhibitors. We first used western blotting to detect single bands at the correct molecular weights for both RIP1 and RIP3 from cochlear samples. Exposure to $106 \mathrm{~dB}$ SPL did not change the band density of RIP1 or RIP3 in total cochlear homogenates $1 \mathrm{~h}$ after exposure (data not shown). Next, the expression of RIP1 and RIP3 in OHCs was examined by immunolabelling surface preparations $1 \mathrm{~h}$ after noise exposure and control preparations. Immunofluorescence of both RIP1 and RIP3 in OHCs was stronger after noise treatment compared with control OHCs (Figures $2 \mathrm{a}$ and b, panel: Noise). Quantitative analysis revealed a significant increase in immunofluorescence, with a control-to-noise ratio of $1: 1.7$ for RIP1 (Figure 2a' $t_{4}=-8.233, P=0.001$ ) and $1: 1.5$ for RIP3 (Figure 2b' $t_{4}=-8.217, P=0.001$ ). In order to determine whether the changes in RIP1 and RIP3 expression occurred only under $106 \mathrm{~dB}$ SPL noise condition that induces permanent deficits, a group of mice was exposed to lower levels of BBN ( $92 \mathrm{~dB} \mathrm{SPL}$ ) resulting in only temporary threshold shifts (TTS). Under this condition, there was no increase in immunofluorescence of RIP1 or RIP3 in OHCs compared with control (data not shown).

As we observed that both necrosis inhibitor $\mathrm{Nec}-1$ and apoptosis inhibitor ZVAD alter the number of necrotic OHCs, we assessed the effect of both compounds on RIP kinases. Treatment with ZVAD significantly enhanced noise-induced RIP1- and RIP3-associated immunofluorescence in OHCs (Figures 2a and b, panel: Noise + ZVAD). Quantification of RIP1- and RIP3-associated immunofluorescence in OHCs confirmed a statistically significant increase (Figure 2a', RIP1: $t_{8}=-3.163, \quad P=0.013 ; \quad$ Figure 2b', RIP3: $t_{8}=-3.92$, $P=0.004)$. On the other hand, treatment with $\mathrm{Nec}-1$ resulted in a reduction of noise-induced RIP1 and RIP3 immunofluorescence (Figures 2a and b, panel: Noise + Nec1). Quantitative analysis of RIP1- and RIP3-associated immunofluorescence in OHCs confirmed a statistically significant decrease (Figure $2 \mathrm{a}^{\prime}, \mathrm{RIP1}: t_{8}=6.058, P<0.001$; Figure $2 b^{\prime}$, RIP3: $t_{8}=4.08, P=0.004$ ).

Noise-induced enhancement of p-AMPK $\alpha$ in OHCs was modulated by RIP1. We previously reported that noise increases the levels of $\mathrm{p}-\mathrm{AMPK} \alpha$ in $\mathrm{OHCs}$, indicating a transient ATP depletion that may contribute to noise-induced necrotic cell death. ${ }^{15}$ We therefore analyzed the relationship between RIP3 and p-AMPK $\alpha$. First, western blotting of cochlear homogenates resulted in a single anti-p-AMPK band for each sample, with no difference in band densities between control and noise-exposed groups (data not shown). However, the levels of $\mathrm{p}-\mathrm{AMPK} \alpha$ significantly increased in OHCs $1 \mathrm{~h}$ after noise exposure, in agreement with our previous studies (Figure 3a, panel: Noise), with a ratio of $1: 3.1$ of control-to-noise samples (Figure $3 b$, $\left.t_{3}=-8.725, P=0.003\right)$. Next, we found that noise-induced $\mathrm{p}$-AMPK $\alpha$ fluorescence in $\mathrm{OHCs}$ decreased when RIP proteins were inhibited by necrosis inhibitor Nec-1 (Figure 3a, panel: Noise $+\mathrm{Nec}-1$; Figure $3 \mathrm{~b}, t_{6}=2.620$, $P=0.04)$. In contrast, treatment with the apoptosis inhibitor ZVAD did not alter the immunofluorescence of $\mathrm{p}-\mathrm{AMPK} \alpha$ in OHCs (Figure 3a, panel: Noise +ZVAD; Figure 3b, $\left.t_{6}=0.673, P=0.53\right)$.

RIP3 siRNA-inhibited noise-induced expression of RIP3 and $\mathrm{p}-\mathrm{AMPK} \alpha$ in OHCs resulting in decreased necrotic $\mathrm{OHC}$ death, but increased the number of apoptotic nuclei. First, local delivery of siRIP3 efficiently suppressed RIP3 expression in OHCs. A decrease in RIP3-associated immunofluorescence was seen in OHCs $72 \mathrm{~h}$ after siRIP3 delivery compared with scrambled-siRNA control (siControltreated ears without noise, Figure 4a). Quantitative analysis of the ratio of RIP3 expression in siControl-treated OHCs to siRIP3-treated OHCs was 1:0.7 (Figure 4a', siControl versus siRIP3: $\left.t_{3}=6.631, P=0.007\right)$. Treatment with siRIP3 alone did not cause conductive hearing loss or sensory hair cell loss $72 \mathrm{~h}$ after the delivery (data not shown).

Next, pretreatment with siRIP3 significantly diminished the noise-induced increase in the levels of RIP3, while treatment with siControl had no effect (Figures $4 b$ and $b^{\prime}$, siControl plus noise versus siRIP3 plus noise: $t_{6}=4.373, P=0.005$ ).

As the inhibition of RIPs by Nec-1 diminishes noise-induced AMPK activation in OHCs, we tested whether siRIP3 treatment also reduces noise-induced activation of AMPK. Immunofluorescence of $\mathrm{p}-\mathrm{AMPK} \alpha$ was weaker in noiseexposed OHCs treated with SiRIP3 than in siControl preparations (Figure 4c). Quantitative analysis of $p-A M P K \alpha$ associated immunofluorescence confirmed the significant decrease with siRIP3 treatment (Figure $4 c^{\prime}, t_{6}=3.800$, $P=0.009$ ).

Furthermore, the downregulation of RIP3 by siRNA resulted in a significant reduction of noise-induced swollen $\mathrm{OHC}$ nuclei (Figure $4 \mathrm{~d}, t_{8}=2.825, P=0.022$ ) and a significant increase in condensed $\mathrm{OHC}$ nuclei $1 \mathrm{~h}$ after noise exposure (Figures $4 \mathrm{~d}$, $\left.t_{8}=-2.523, P=0.036\right)$.

ZVAD, but not Nec-1 or siRIP3 treatment, inhibited noise-induced caspase-8 activation in OHCs. First, western blotting using total cochlear homogenates from control without noise and noise-exposed mice showed no difference in the band density of cleaved caspase-8 (CC8) (data not shown). However, noise treatment induced a significant increase in $\mathrm{CC} 8$ in $\mathrm{OHCs}$ on surface preparations (Figure 5a, panel: Noise; Figure $5 a^{\prime}, t_{4}=-6.523$, $P=0.003$ ). Next, treatment with local delivery of ZVAD significantly reduced the level of noise-induced CC8 (Figure 5a, panel: Noise+ZVAD; Figure $5 a^{\prime}, t_{8}=2.766$, $P=0.025$ ). In contrast, neither Nec-1 (Figure 5a, panel: Noise + Nec-1; Figure $\left.5 b, t_{8}=1.072, P=0.315\right)$ nor siRIP3 treatment (Figure $5 b, t_{3}=0.355, P=0.746$ ) affected the levels of CC8.

Silencing RIP3 increased noise-induced activation of caspase-9 and translocation of EndoG into the nuclei of OHCs. As treatment with either necrosis inhibitor $\mathrm{Nec}-1$ or 
a
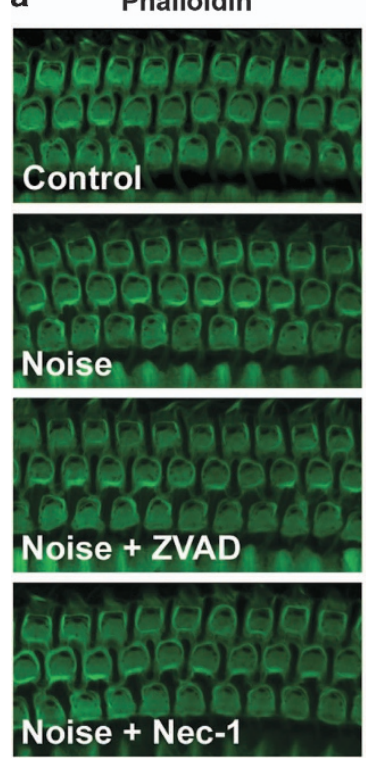

b Phalloidin
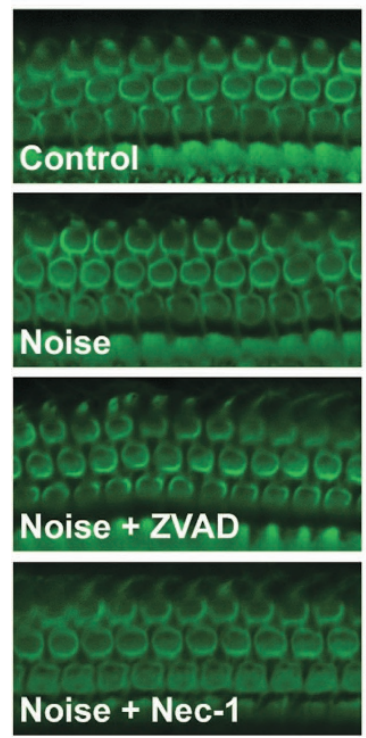

RIP1
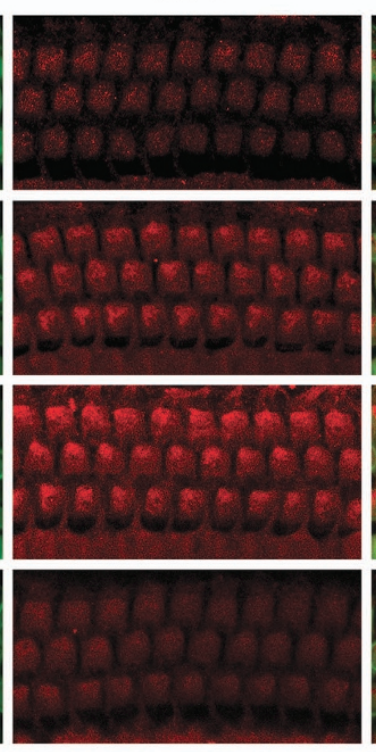

RIP3
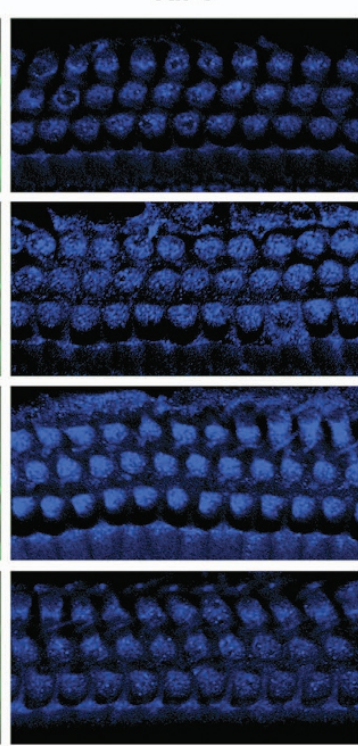

Merged
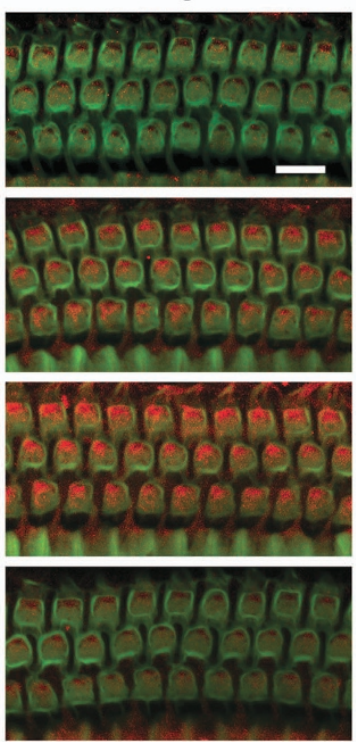

Merged
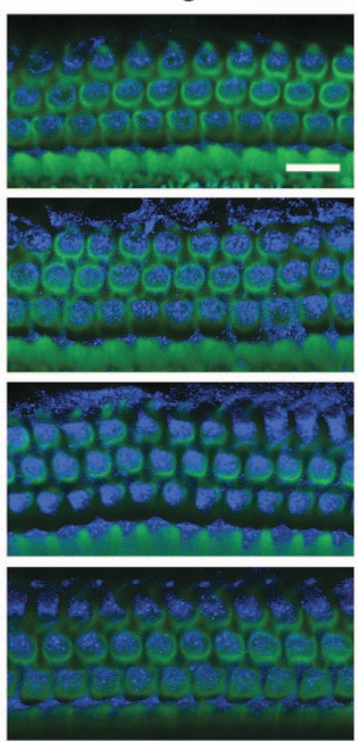

$\mathbf{a}^{\prime}$
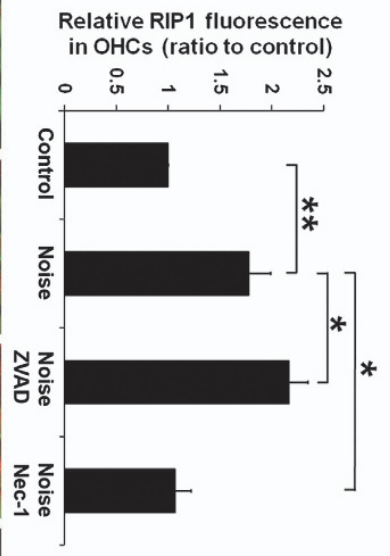

$\mathbf{b}^{\prime}$

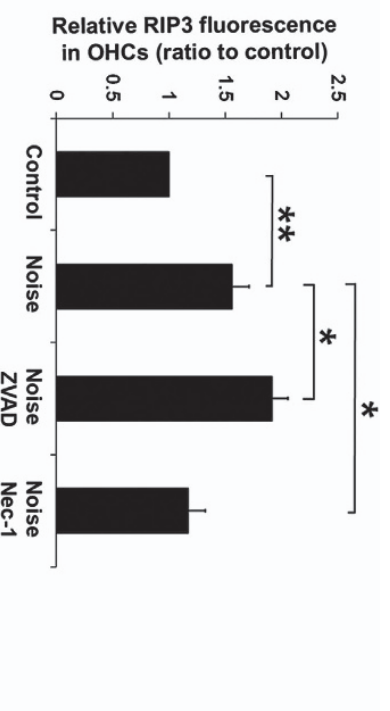

Figure 2 Noise-induced elevation of RIP1 and RIP3 levels in sensory hair cells is modulated by treatment with ZVAD or Nec-1. (a) Sensory hair cells from the upper basal region of the cochlear epithelium show changes in RIP1-associated immunofluorescence. Control: baseline levels of RIP1 without noise exposure. Noise: RIP1-associated immunofluorescence is stronger $1 \mathrm{~h}$ after $106 \mathrm{~dB}$ SPL noise. Noise + ZVAD: treatment with ZVAD via local delivery increases noise-induced RIP1 immunofluorescence. Noise + Nec-1: treatment with Nec-1 via local delivery decreases noise-induced RIP1-associated immunofluorescence. Green: phalloidin labeling of sensory hair cells; red: RIP1. Images are representative of five individual ears for each condition. Scale bar $=10 \mu \mathrm{m}$. (a') Quantification of relative RIP1-associated immunofluorescence in OHCs reveals a significant enhancement $1 \mathrm{~h}$ after $106 \mathrm{~dB}$ SPL noise exposure. Noise-induced elevation of RIP1 levels is further promoted by treatment with ZVAD and suppressed by Nec-1 treatment via local delivery. Data are presented as means + S.D.; $n=5$ for each condition; ${ }^{*} P<0.05,{ }^{* \star} P<0.001$. (b) Sensory hair cells from the upper basal turn of the cochlear epithelium show alteration of RIP3-associated immunofluorescence. Control: baseline levels of RIP3 immunofluorescence without noise exposure. Noise: RIP3 immunofluorescence increases $1 \mathrm{~h}$ after $106 \mathrm{~dB}$ SPL noise exposure. Noise + ZVAD: local delivery of ZVAD increases noise-induced RIP3-associated fluorescence. Noise + Nec-1: treatment with Nec-1 via local delivery attenuates noise-induced enhancement of RIP3 intensity. Green: phalloidin labeling of sensory hair cells; blue: RIP3. Images are representative of five individual ears for each condition. Scale bar $=10 \mu \mathrm{m}$. ( $\mathbf{b}^{\prime}$ ) Quantification of RIP3-associated relative fluorescence in OHCs confirms a significant enhancement $1 \mathrm{~h}$ after $106 \mathrm{~dB}$ SPL noise exposure. Noise-induced RIP3-associated immunofluorescence is further increased by ZVAD treatment and decreased by Nec-1 treatment. Data are presented as means + S.D.; $n=5$ for each condition; ${ }^{*} P<0.05,{ }^{*} P<0.001$

siRIP3 increased the number of apoptotic OHCs without altering the levels of CC8 after noise exposure, the levels of alternate apoptotic mediators (CC9 and EndoG translocation into nuclei) after treatment with siRIP3 were analysed. The amount of $\mathrm{CC} 9$ in $\mathrm{OHCs}$ significantly increased $1 \mathrm{~h}$ after noise compared with that of control mice without noise exposure (Figure 5c, panel: siControl + Noise; Figure $5 c^{\prime}$, $\left.t_{3}=-7.442, P=0.005\right)$. This noise-induced activation of CC9 was potentiated by treatment with siRIP3 compared with siControl mice (Figure 5c, panel: siRIP3+Noise; 
a
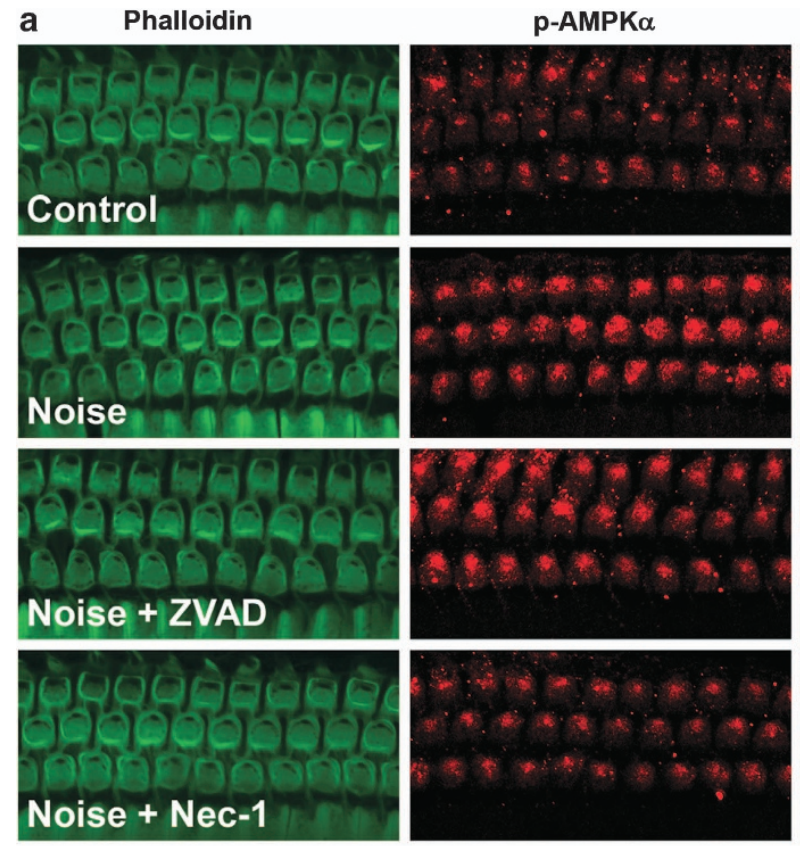

b

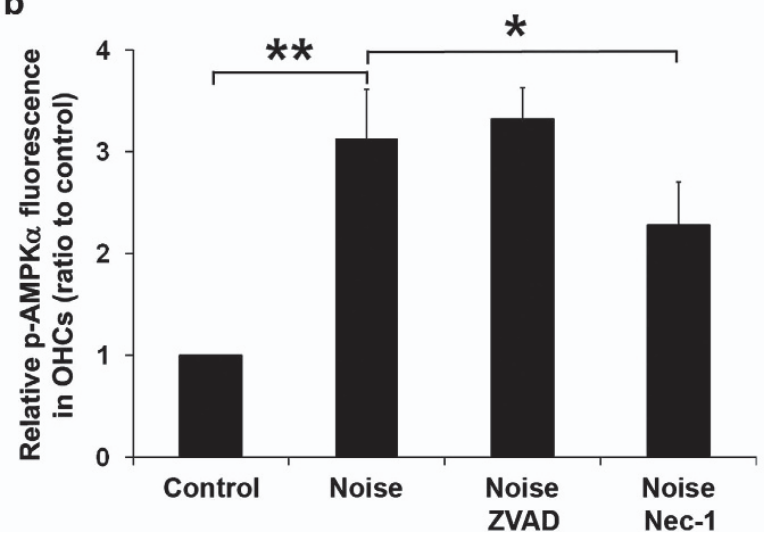

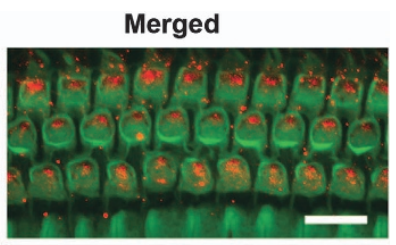
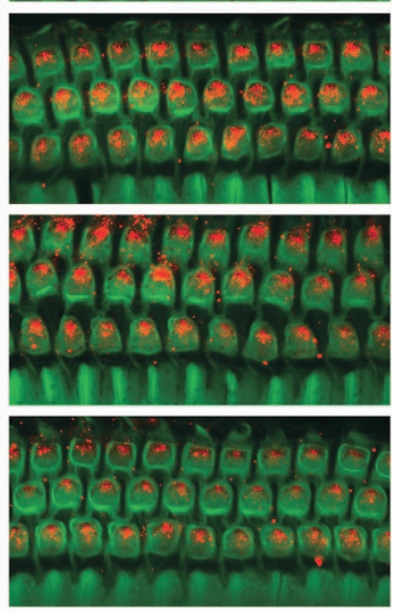

Figure 3 Noise-induced p-AMPK $\alpha$ expression in sensory hair cells is modulated by Nec-1 treatment. (a) p-AMPK $\alpha$ fluorescence in sensory hair cells. Control: baseline levels of $p$-AMPK $\alpha$-associated immunofluorescence without noise exposure. Noise: $p$-AMPK $\alpha$ fluorescence increases $1 \mathrm{~h}$ after $106 \mathrm{~dB}$ SPL noise exposure. Noise + ZVAD: treatment with ZVAD via local delivery does not alter noise-induced p-AMPK $\alpha$ levels. Noise + Nec-1: treatment with Nec-1 via local delivery attenuates the noise-induced elevation of $p$-AMPK $\alpha$ fluorescence. Green: phalloidin labeling of sensory hair cells; red: $p$-AMPK $\alpha$. Images are taken from the upper basal turn and are representative of five individual ears for each condition. Scale bar $=10 \mu \mathrm{m}$. (b) Quantification of relative $\mathrm{p}$-AMPK $\alpha$-associated fluorescence in OHCs confirms a significant increase after $106 \mathrm{~dB}$ SPL noise exposure. This increase is blocked by Nec-1 treatment but does not change with ZVAD treatment. Data are presented as means + S.D.; $n=5$; ${ }^{\star} P<0.05$, ${ }^{* *} P<0.001$

Figure $\left.5 \mathrm{c}, t_{6}=-2.603, P=0.041\right)$. Finally, we assessed localization of EndoG in OHCs in three experimental groups (siControl, siControl plus noise, and siRIP3 plus noise) on cochlear surface preparations. EndoG was localized to the cytosol of OHCs without nuclear labeling in mice treated with siControl. One hour after noise exposure, strong EndoG immunofluorescence appeared in a few $\mathrm{OHC}$ nuclei in the lower basal turn (Figure 6a, panel: siControl + Noise, arrows), while with SiRIP3 treatment numbers of OHCs with translocation of EndoG to the nuclei significantly increased (Figure 6a, panel: siRIP3 + Noise; Figure 6b, $\left.t_{6}=-4.099, P=0.006\right)$.

Treatment with a combination of siRIP3 and ZVAD additively protected against NIHL and $\mathrm{OHC}$ death. We first examined the abilities of the caspase inhibitor ZVAD or
siRIP3 to prevent NIHL. Two weeks after $98 \mathrm{~dB}$ SPL noise exposure, the auditory brainstem response (ABR) threshold shifts of controls without noise exposure, noise-only, noise plus DMSO (vehicle of ZVAD), and noise plus ZVAD treatment groups via intraperitoneal (IP) injection differed significantly at $8 \mathrm{kHz}$ (Figure $7 \mathrm{a}, \mathrm{F}_{3,20}=12.344, P<0.001$ ), $16 \mathrm{kHz}$ (Figure $8 \mathrm{a}, \mathrm{F}_{3,20}=43.629, P<0.001$ ) and $32 \mathrm{kHz}$ (Figure 7a, $F_{3,20}=93.528, P<0.001$ ). Tukey's multiple comparisons test showed that treatment with ZVAD significantly reduced $\mathrm{NIHL}$ at both $16 \mathrm{kHz}(P=0.042)$ and $32 \mathrm{kHz}(P=0.028)$, but not at $8 \mathrm{kHz}(P=0.845)$ compared with noise-exposed mice not treated with ZVAD. Conversely, the delivery of SiRIP3 alone did not reduce noise-induced threshold shifts at any of the measured frequencies by oneway ANOVA analysis (Figure 7b). Importantly, the ABR 
threshold shifts of the three groups (noise plus DMSO with siControl, noise plus ZVAD with siControl, and noise plus ZVAD with siRIP3) were significantly different at both $16 \mathrm{kHz}$ $\left(F_{2,12}=19.971, \quad P<0.001\right)$ and $32 \mathrm{kHz} \quad\left(F_{2,12}=20.179\right.$, $P<0.001)$, but not at $8 \mathrm{kHz}\left(F_{2,12}=3.093, P=0.083\right)$. The combined treatment of ZVAD and siRIP3 significantly reduced noise-induced $\mathrm{ABR}$ threshold shifts at both $16 \mathrm{kHz}$ (Figure 7c, $P=0.01$ ) and $32 \mathrm{kHz}$ (Figure 7c, $P=0.014$ ) compared with treatment with ZVAD alone. Two-way ANOVA analysis showed no significant interaction between ZVAD and siRIP3 treatments at either $16 \mathrm{kHz}(P=0.093)$ or $32 \mathrm{kHz}$ $(P=0.546)$. a
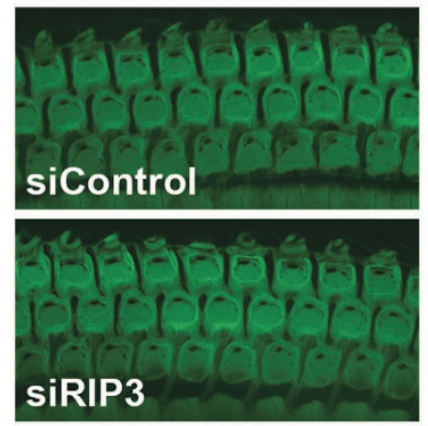

b

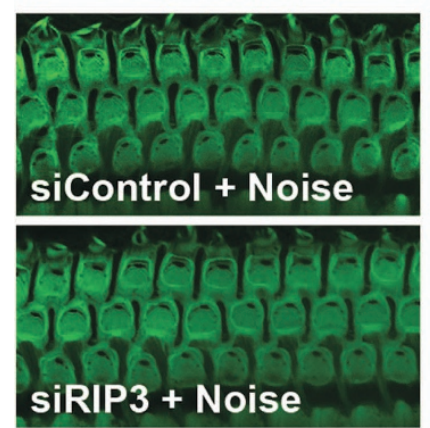

C
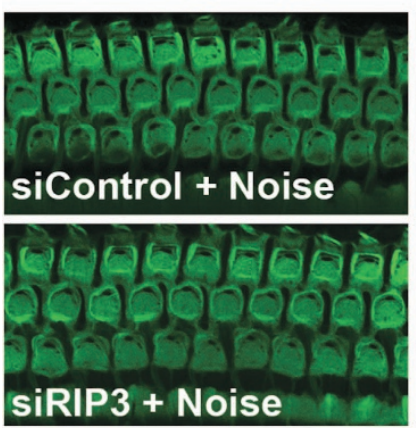

RIP3
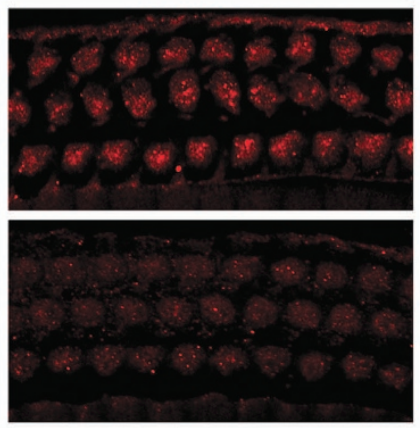

RIP3
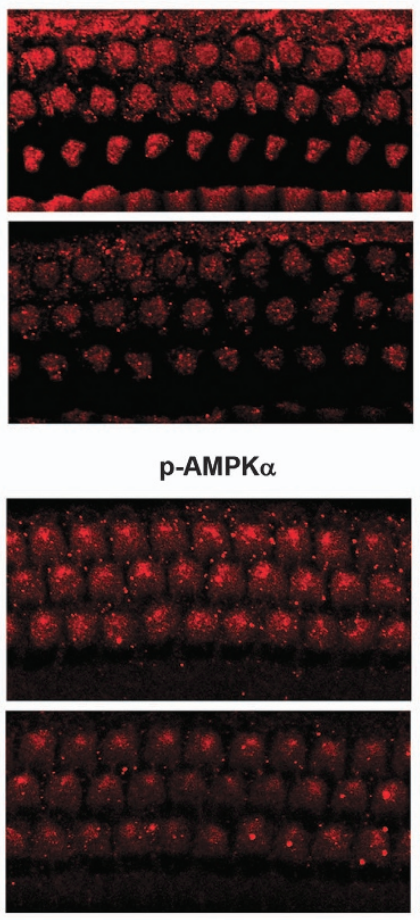

Merged
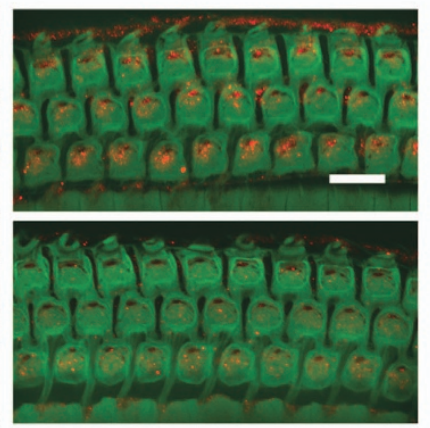

Merged

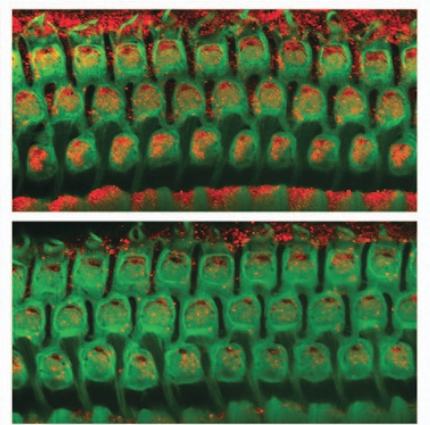

Merged

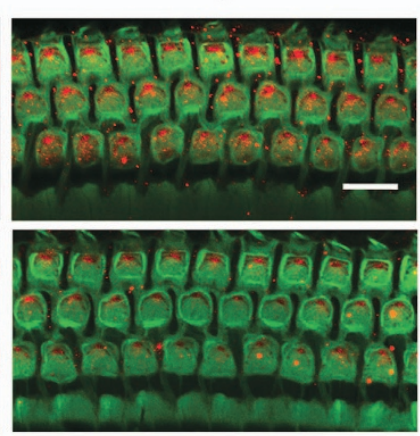

$\mathbf{a}^{\prime}$

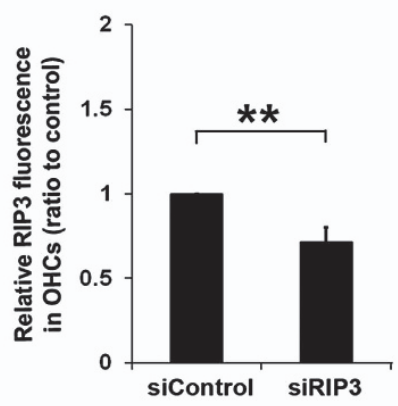

$b^{\prime}$

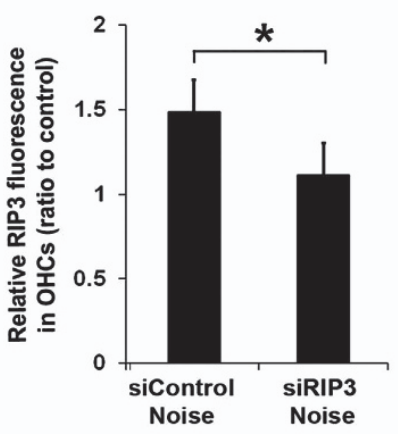

$c^{\prime}$

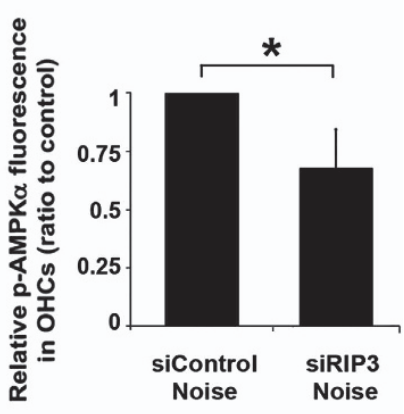

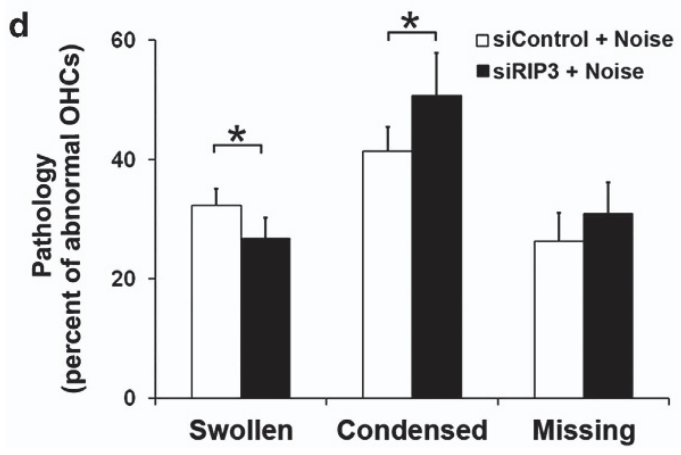


Next, $\mathrm{OHC}$ loss was quantified along the entire length of the cochlear epithelium. There was a significant difference within the four groups of control without noise, noise-only, noise plus ZVAD with siControl, and noise plus ZVAD with siRIP3 treatment (Figure $7 \mathrm{~d}, F_{2,9}=21.157, P<0.001$ ). Noise treatment induced a significantly higher amount of $\mathrm{OHC}$ loss compared with control mice $(P<0.001)$. Treatment with ZVAD alone did not have a significant effect on noise-induced $\mathrm{OHC}$ loss. However, combined treatment of ZVAD plus siRIP3 significantly reduced noise-induced $\mathrm{OHC}$ losses compared with treatment with ZVAD $(P=0.005)$. In addition, noiseinduced $\mathrm{OHC}$ losses started at $3.0 \mathrm{~mm}$ from the apex with a gradient of increased loss toward the base. At $5.5 \mathrm{~mm}$ from the apex, loss of OHCs was complete. With the combined treatment of ZVAD and siRIP3, loss of OHCs began closer to the base. The trend analysis showed a significant difference in cubic components $(P=0.013)$.

\section{Discussion}

The salient findings of this study are that noise-induced death of OHCs occurs as a balance between apoptosis and necrosis, regulated by caspase- 8 and RIP kinases. On one hand, the pan-caspase inhibitor ZVAD blocks noise-induced activation of caspase-8 and reduces apoptosis in OHCs, while stimulating an increase in levels of RIP1 and RIP3 to enhance $\mathrm{OHC}$ necrosis. On the other hand, treatment with either necrosis inhibitor Nec-1 or SiRIP3 diminishes the expression of RIPs, blocks noise-induced enhancement of p-AMPK, a marker of cellular energy depletion, and reduces $\mathrm{OHC}$ necrosis, while increasing $\mathrm{OHC}$ apoptosis by activating caspase-9 and promoting EndoG translocation to the nuclei of OHCs. Consequently, treatment with ZVAD significantly reduces noise-induced threshold shifts, but combined treatment with SiRIP3 provides additive protective effects on both hearing and hair cell loss. These results demonstrate molecular signals of necrotic $\mathrm{OHC}$ death and suggest that effective pharmacological protection against $\mathrm{NIHL}$ needs to address both apoptotic and necrotic $\mathrm{OHC}$ death pathways.

Apoptosis is a highly regulated process mediated by caspases and characterized by cellular shrinkage, chromatin condensation, and DNA degradation. In contrast, necrosis is characterized by cellular swelling, plasma membrane rupture, and the subsequent loss of intracellular contents. ${ }^{25}$ Based on such morphological criteria, we demonstrate both apoptosis and necrosis in OHCs following traumatic noise exposure, supporting earlier reports in the literature. ${ }^{15,23}$ The fact that the increase in levels of RIP1 and RIP3 in OHCs is seen after exposure to traumatic noise but not after lower-level noise strongly suggests that cell death triggered by the noiseinduced stress involves RIP kinase family members, a notion in agreement with similar observations in ischemic injury, viral infection, and retinal detachment-induced photoreceptor necrosis. ${ }^{4,9,26}$

The increase in the levels of p-AMPK $\alpha$ in OHCs induced by noise implies depletion of cellular ATP, in accordance with our earlier observations. ${ }^{15}$ Such ATP depletion may cause cell death via either apoptosis or necrosis. ${ }^{11,27}$ In our case, silencing RIP3 or blocking RIP1 with Nec-1 treatment reduces noise-induced activation of AMPK $\alpha$ and also lowers $\mathrm{OHC}$ necrosis, suggesting that RIP-kinases are involved in initiating necrosis through a pathway that alters ADP/ATP exchange, leading to a lethal decline in intracellular ATP. ${ }^{8-11}$ As RIP1/ RIP3-dependent necrosis can be induced by engagement of death receptors such as TNFR-1 and TNF receptor superfamily member 6 (FasR) to form a death signaling complex with caspase-8, ${ }^{5,6}$ we may speculate that activation of RIP1/ RIP3 in OHCs by noise occurs through stimulation of death receptors. This idea is supported by evidence that TNF $\alpha$ is produced by $\mathrm{OHCs}$, supporting cells, and fibrocytes in the spiral ligament following a variety of insults including noise exposure, excessive vibration, and treatment with cisplatin. ${ }^{28-32}$

Apoptosis is also evident after noise exposure. Treatment with ZVAD blocks noise-induced activation of caspase-8 and reduces NIHL, supporting the involvement of CC8 in apoptotic $\mathrm{OHC}$ death. ${ }^{20-24}$ However, necrotic and apoptotic pathways do not proceed independently but are reciprocally regulated. In agreement with such a notion, our novel observation is that inhibition of caspase-8 by ZVAD increases the levels of RIP1 and RIP3 in OHCs. Such inhibition is essential for the activities of both RIP1 and RIP3 kinases ${ }^{9,33}$ and induce RIP1/RIP3-dependent necrosis, demonstrating that inhibition of apoptosis may lead to an increase in necrosis signaling molecules.

The fact that both Nec-1 treatment and RIP3 silencing reduces $\mathrm{OHC}$ death establishes that RIPs are crucial regulators of controlled $\mathrm{OHC}$ necrosis. Interestingly, although this treatment increases the number of apoptotic OHCs, it does not alter CC8 levels, suggesting that these necrosis

\footnotetext{
Figure 4 siRIP3 prevents noise-induced RIP3 and p-AMPK $\alpha$ expression in OHCs. (a) RIP3-associated immunofluorescence in sensory hair cells $72 \mathrm{~h}$ after siRIP3 delivery. siControl: Baseline levels of RIP3 immunofluorescence after treatment with scrambled siRNA. siRIP3: treatment with siRIP3 diminishes RIP3-asssociated immunofluorescence. Green: phalloidin labeling of sensory hair cells; red: RIP3. (a') Quantification of relative RIP3-associated immunofluorescence in OHCs confirms a significant decrease with siRIP3 treatment. Data are presented as means + S.D.; $n=6 ;{ }^{* \star} P<0.01$. (b) siControl + Noise: treatment with scrambled siRNA (siControl) does not affect the noise-induced increase in RIP3 immunofluorescence $1 \mathrm{~h}$ after $106 \mathrm{~dB}$ SPL noise exposure. siRIP3 + Noise: treatment with siRIP3 diminishes noise-induced enhancement of RIP3 immunofluorescence $1 \mathrm{~h}$ after $106 \mathrm{~dB}$ SPL noise exposure. Images in both $\mathbf{a}$ and $\mathbf{b}$ are taken from the upper basal turn and are representative of five individual ears for each condition. Scale bar $=10 \mu \mathrm{m}$. ( $\left.\mathbf{b}^{\prime}\right)$ Quantification of relative RIP3 fluorescence in OHCs confirms that noise-induced elevation of RIP3 is significantly attenuated by siRIP3 treatment. Data are presented as means + S.D.; $n=5$; ${ }^{\star} P<0.05$. (c): Noise-induced (106 dB SPL) p-AMPK $\alpha$-associated immunofluorescence in sensory hair cells $1 \mathrm{~h}$ after the exposure (siControl + Noise) is blocked by siRIP3 treatment (siRIP3 + Noise). Green: phalloidin labeling of sensory hair cells; red: $p$-AMPK $\alpha$. Images are taken from the upper basal turn of the cochlear spiral and are representative of five individual ears for each condition. Scale bar $=10 \mu \mathrm{m}$. (c $\left.{ }^{\prime}\right)$ Quantification of relative $\mathrm{p}$-AMPK $\alpha$-associated immunofluorescence in OHCs from the upper basal turn confirms a significant decrease in noise-induced $p$-AMPK $\alpha$ immunofluorescence. Data are presented as means + S.D.; $n=5$; ${ }^{*} P<0.05$. (d) Quantification of noise-induced swollen, condensed, or missing OHC nuclei, as determined by PI labeling, in the lower basal turn after siRIP3 treatment shows a decrease in swollen nuclei but an increase in condensed nuclei, while there is no difference in the amount of missing OHCs $1 \mathrm{~h}$ after the noise exposure. Data are presented as means + S.D.; $n=5 ;{ }^{*} P<0.05$
} 
a
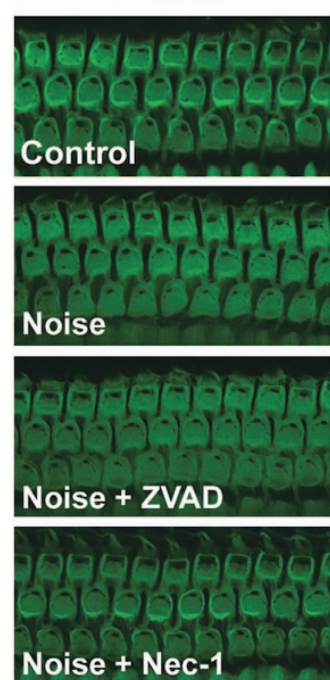

b

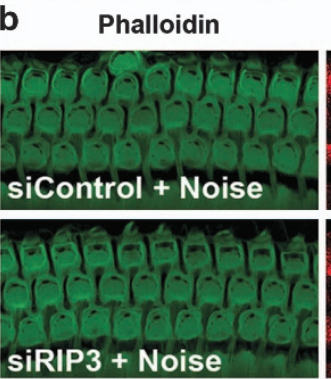

c
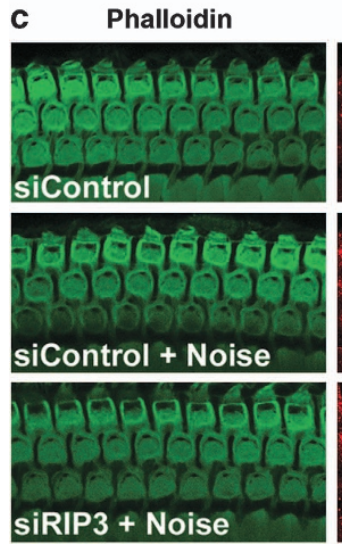

cc8
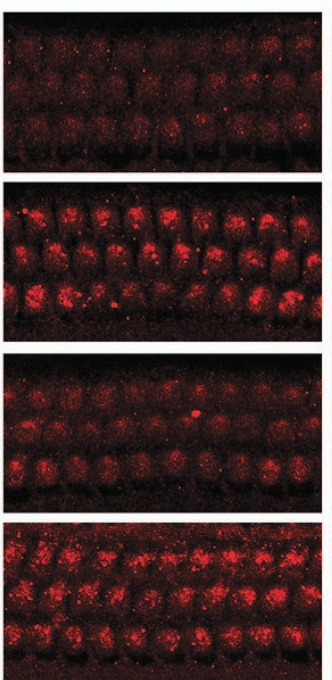

CC8
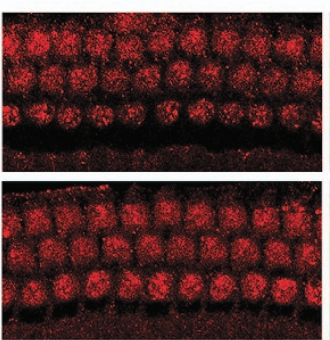

Cc9
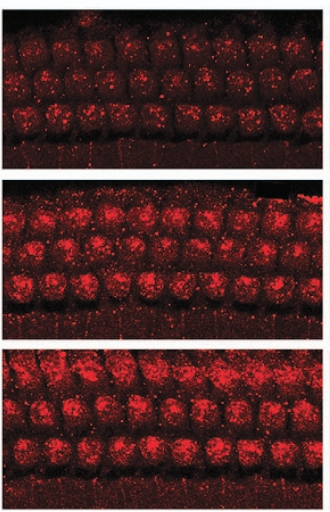

Merged
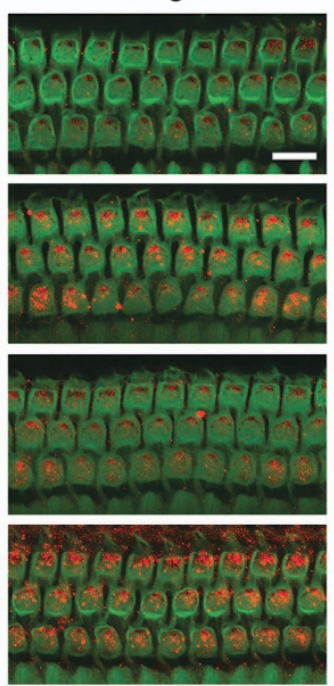

Merged
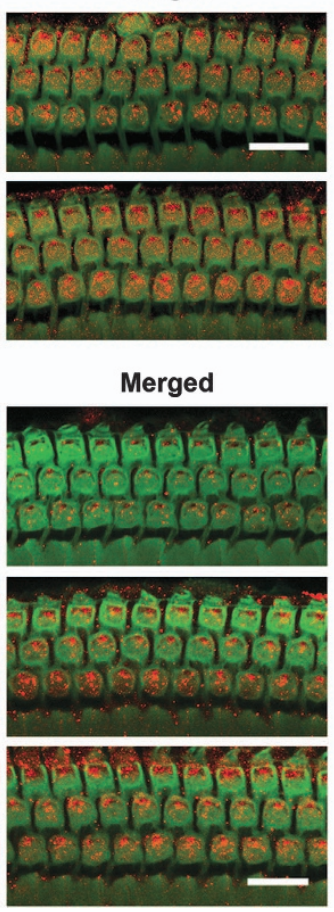

$\mathbf{a}^{\prime}$
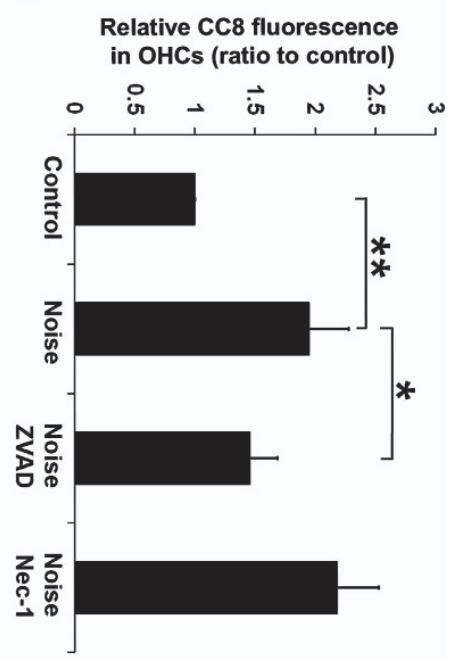

C' Relative CC9 fluorescence in OHCs (ratio to siControl)

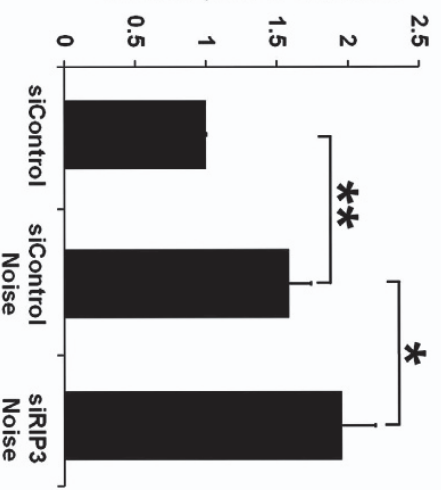

Figure 5 Noise-induced elevation in CC8 is inhibited by ZVAD, but not by Nec-1 or siRIP3 treatment, while noise-induced activation of CC9 in sensory hair cells increases by pretreatment with siRIP3. (a) CC8-associated immunofluorescence in the cochlear epithelium. Control: baseline levels of CC8 without noise exposure. Noise: immunofluorescence associated with CC8 increases $1 \mathrm{~h}$ after $106 \mathrm{~dB}$ SPL noise exposure. Noise + ZVAD: treatment with ZVAD via local delivery inhibits noise-induced elevation of CC8. Noise + Nec-1: treatment with local delivery of Nec-1 does not influence levels of noise-induced CC8. Green: phalloidin labeling of sensory hair cells; red: CC8. Images are taken from the upper basal turn and are representative of five ears for each condition. Scale bar $=10 \mu \mathrm{m}$. (a') Quantification of CC8-associated fluorescence in OHCs confirms a significant increase after noise exposure. Noise-induced CC8 is blocked by local delivery of ZVAD but is not influenced by Nec-1 treatment. Data are presented as means + S.D.; $n=5 ;{ }^{*} P<0.05,{ }^{* *} P<0.001$. (b) There is no difference in CC8-associated immunofluorescence between $106 \mathrm{~dB}$ SPL noise-exposed siRIP3 and siControl samples. (c) Induction of CC9 by noise exposure is increased by treatment with siRIP3. Green: phalloidin labeling of sensory hair cells; red: CC8 or CC9. Images are taken from the upper basal turn and are representative of five ears for each condition. Scale bar $=10 \mu \mathrm{m}$. (c') Quantification of CC9-associated immunofluorescence in OHCs confirms a significant increase after siRIP3 treatment. Data are presented as means + S.D.; $n=5 ;{ }^{*} P<0.05,{ }^{* \star} P<0.001$

regulators must modulate other apoptotic signaling pathways. Activation of caspase- 9 and EndoG nuclear translocation are the likely targets for RIP3 regulation and the cause of increases in the number of apoptotic OHCs after treatment with siRIP3. This observation is in agreement with and expands upon earlier reports of noise-induced increases in cleaved caspase-9 (CC9) and EndoG translocation. ${ }^{21,23,24,34} \mathrm{~A}$ shift of cell death pathways is also a reasonable explanation for the failure of only silencing RIP3 to confer a protective effect against NIHL. Finally, the balance between apoptotic and necrotic signal transduction 
a
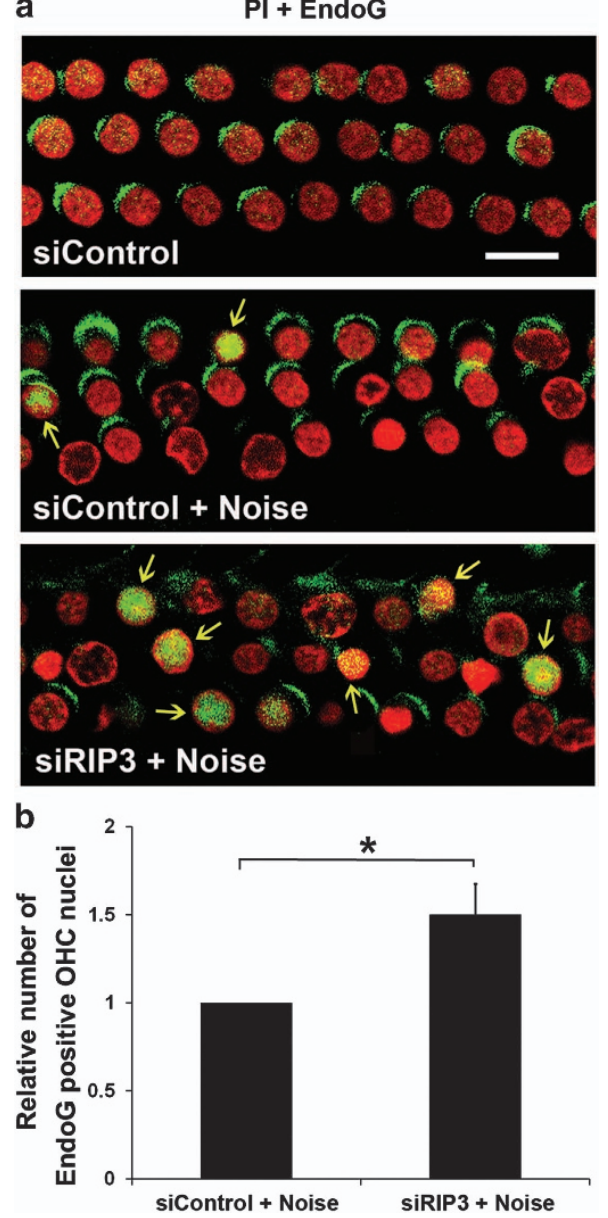

Figure 6 siRIP3 treatment increases noise-induced Endo G translocation into nuclei of OHCs. (a) Immunolabeling of EndoG in OHCs of cochlear epithelia. siControl: EndoG is localized to the cytosol of OHCs. siControl + Noise: EndoG is found in a few nuclei $1 \mathrm{~h}$ after $106 \mathrm{~dB}$ SPL noise exposure (arrows). siRIP3 + Noise: silencing RIP3 increases noise-induced EndoG translocation into nuclei (arrows). Red: PI staining for nuclei; green: EndoG. Images are taken from the cochlear lower basal turn and are representative of five ears for each condition. (b) Quantification of OHCs with EndoGpositive nuclei in the lower basal turn confirms a significant increase with siRIP3 treatment. Data are presented as means + S.D.; $n=5 ;{ }^{*} P<0.05$

pathways in $\mathrm{OHC}$ death is strongly supported by the additive protection against NIHL and hair cell loss through the combined treatment of ZVAD and siRIP3.

In summary, our results demonstrate that noise may cause both an increase in RIP kinases and activation of caspase-8 (Figure 8). The activation of caspase-8 leads to apoptotic hair cell death, whereas the increase in RIP1 and RIP3 aggravates cellular energy depletion and results in necrotic cell death. Importantly, a balance exists between apoptotic and necrotic pathways. Inhibition of CC8 blocks apoptotic cell death but stimulates an increase in RIP1 and RIP3, and OHC necrosis. In contrast, inhibition of RIP1 and RIP3 diminishes the expression of RIP1 and RIP3, but stimulates the activation of caspase-9 and increases EndoG translocation into the nuclei of $\mathrm{OHCs}$, resulting in apoptotic cell death. As a consequence of this balance, the prevention of NIHL requires pharmacological combination treatment to address both apoptotic and necrotic pathways.

\section{Materials and Methods}

Animals. Male CBA/J mice at 12 weeks of age (Harlan-Sprague-Dawley) had free access to water and a regular mouse diet (Purina 5025) and were kept at $22 \pm 1^{\circ} \mathrm{C}$ under a standard $12: 12 \mathrm{~h}$ light-dark cycle to acclimate for 1 week before the experiments. All research protocols were approved by the Institutional Animal Care and Use Committee at the Medical University of South Carolina (MUSC). Animal care was under the supervision of the Division of Laboratory Animal Resources at MUSC.

Noise exposure. Four separate stainless steel wire cages $(9 \mathrm{~cm} \times 9 \mathrm{~cm} \times 9$ $\mathrm{cm}$ each) were located in a sound-exposure chamber. The sound-exposure chamber was fitted with a loudspeaker (model $2450 \mathrm{H}$; JBL, Northridge, CA, USA) driven by a power amplifier (model XLS 202D; Crown Audio, New York, NY, USA) fed from a CD player (model CD-200; Tascam TEAC Professional, Tokyo, Japan). Audio $C D$ sound files were created and equalized with audio editing software (Audition 3; Adobe System, Inc., San Jose, CA, USA). Sound levels were calibrated with a sound level meter (model 1200; Quest Technologies, Oconomovoc, WI, USA) at multiple locations within the chamber to ensure uniformity of the sound field among the four cages, and were measured before and after exposure to ensure stability.

In order to induce permanent threshold shifts (PTS), animals were exposed to BBN (2-20 kHz) for $2 \mathrm{~h}$ at 98 or $106 \mathrm{~dB}$ SPL. Exposure to $106 \mathrm{~dB}$, which results in severe inner ear damage (losses of both OHCs and IHCs 2 weeks after noise exposure), was used for the experiments on cell death pathways; a less-severe insult by $98 \mathrm{~dB}$ (only loss of OHCs, but not IHCs 2 weeks after noise exposure) was chosen for the experiments on protection against NIHL (Figure 8) in order to allow for a more sensitive detection of potential protective effects. TTS were induced by lowering the exposure intensity to $92 \mathrm{~dB}$ SPL. Before mice were exposed in the sound chamber for induction of the TTS, the sound intensity of the environment surrounding the cages was $65 \mathrm{~dB}$ as measured with a sound level meter (model 1200; Quest Technologies). In general, four mice (one mouse per cage) were exposed at the same time. If compounds had to be delivered to the middle ear cavity immediately after exposure, only one mouse was placed into the chamber at a time. Control mice were kept in silence (without turning on the loudspeaker) within the same chamber and cages for $2 \mathrm{~h}$.

Auditory brainstem response. Mice were anesthetized with an IP injection of ketamine $(100 \mathrm{mg} / \mathrm{kg})$ and xylazine $(10 \mathrm{mg} / \mathrm{kg})$, and then placed in a sound-isolated and electrically shielded sound booth (Kahntact, Hillside, IL, USA). Body temperature was monitored and maintained near $37^{\circ} \mathrm{C}$ with a heating pad. Acoustic stimuli were delivered monaurally to a Beyer earphone attached to a customized plastic speculum inserted into the ear canal. Sub-dermal electrodes were inserted at the vertex of the skull, under the left ear, and under the right ear (ground). ABRs were measured at 8,16 , and $32 \mathrm{kHz}$. Tucker Davis Technology System III hardware and SigGen/Biosig software (Alachua, FL, USA) were used to present the stimuli ( $15 \mathrm{~ms}$ duration tone bursts with $1 \mathrm{~ms}$ rise-fall time) and record the response. Up to 1024 responses were averaged for each stimulus level. Thresholds were determined for each frequency by reducing the intensity in $10 \mathrm{~dB}$ increments and then in $5 \mathrm{~dB}$ steps near threshold until no organized responses were detected. Thresholds were estimated between the lowest stimulus level where a response was observed and the highest level without response. All ABR measurements were conducted by the same experimenter. The ABR thresholds were assigned by an expert who was blinded to the treatment conditions.

Intratympanic delivery of siRNA. siRIP3 (Invitrogen, Carlsbad, CA, USA, s80754) or siControl (Invitrogen) was locally delivered through intratympanic application as previously described. ${ }^{35}$ Briefly, after anesthesia, a retroauricular incision was made to approach the temporal bone. The otic bulla was identified ventral to the facial nerve and a shallow hole was made in the thin part of the otic bulla with a 30-G needle and enlarged with a dental drill to a diameter of $2 \mathrm{~mm}$ in order to visualize the round window. A customized sterile Micro Medical tube was inserted into the hole just above the round window to slowly deliver $10 \mu \mathrm{l}(0.6 \mu \mathrm{g})$ of siRIP3 or siControl. After the siRNA delivery, the hole was covered with surrounding muscle and glued with tissue adhesive. Finally, the skin incision was closed with tissue adhesive and the mouse was kept in the surgical position for $1 \mathrm{~h}$. Seventy-two hours after siRNA delivery, the animals were exposed to BBN or kept in silence in the noise chamber for $2 \mathrm{~h}$.

Intratympanic delivery of ZVAD and Nec-1 for immunofluorescent assay. Stock solutions of the pan-caspase inhibitor Z-VAD-FMK (ZVAD) 

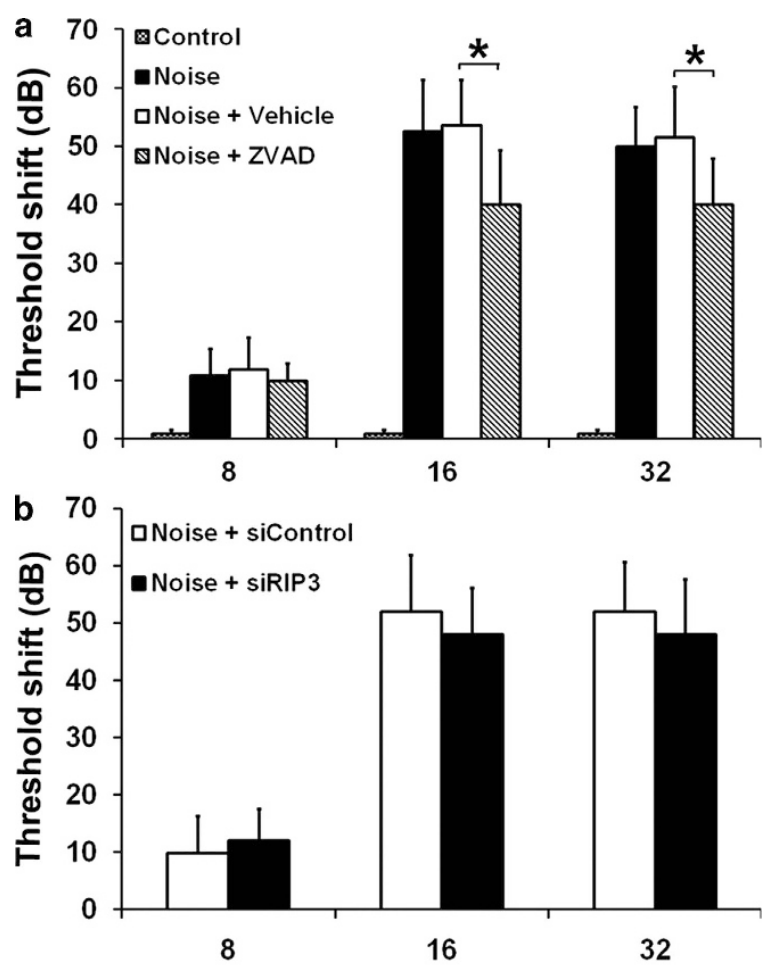

(R\&D Systems, Minneapolis, MN, USA, FMK001) and Nec-1 (Sigma-Aldrich, St. Louis, MO, USA, n9037) were made in DMSO. Immediately before use, each stock solution was diluted in $0.9 \%$ saline. The final concentrations of ZVAD and Nec-1 were $100 \mu \mathrm{M}$ and $300 \mu \mathrm{M}$, respectively, based on the highest concentration without hair cell damage in mouse explant cultures. These concentrations may not be the optimal concentration for the local delivery of ZVAD and Nec-1 to the middle ear of the adult mice. Surgical procedures as described above requiring $\sim 15 \mathrm{~min}$ were conducted immediately following noise exposure and the experimental solution was delivered to fill the otic bulla of the left ear fully. Control mice underwent the same surgical procedure with delivery of identical concentrations of vehicle solution, DMSO diluted in saline, also to the left ear. One hour after noise exposure, the animals were euthanized and the temporal bones were removed to dissect the cochlea for the immunofluorescence assay.

ZVAD IP administration for protection against NIHL. Only in the experiments investigating the protective effects of ZVAD was the treatment administrated via IP injection. ZVAD is an irreversible inhibitor of the caspase family proteases and is commonly used to block caspase-dependent apoptosis. ZVAD was dissolved in DMSO at a stock solution $(10 \mathrm{mg} / \mathrm{ml})$ and stored at $-20^{\circ} \mathrm{C}$. The stock solution of ZVAD was diluted immediately before being injected into animals. Each animal received a total of five IP injections of ZVAD at a dose of $1.5 \mathrm{mg} / \mathrm{kg}$ per injection based on the conditions reported from the literature. ${ }^{36,37}$ Three of the injections were given once per day at 1 day before, and 1 and 2 days after noise exposure. In addition, on the day of noise exposure, the animals received two injections: one immediately before and one after noise exposure. Control animals received the same volume (identical concentration) of DMSO vehicle solution at a concentration of $0.15 \mathrm{ml} / \mathrm{kg}$ on the same schedule.

Surface preparations and DAB staining of cochlear epithelia for sensory hair cell counts. The procedure of surface preparation and diaminobenzidine (DAB) staining of cochlear epithelia was followed as previously described. ${ }^{15}$ Briefly, the temporal bones were removed immediately following euthanasia and perfused through the cochlear scala media with a solution of $4 \%$

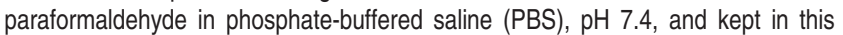
fixative overnight at $4{ }^{\circ} \mathrm{C}$. The cochleae were then rinsed in PBS. Before decalcification in a $4 \%$ solution of sodium EDTA (adjusted with $\mathrm{HCl}$ to $\mathrm{pH} 7.4$ ), the apical otic capsule was removed from each cochlea. The EDTA solution was changed daily for 3 days and maintained at $4{ }^{\circ} \mathrm{C}$. Following decalcification, the cochleae were placed into $3 \%$ hydrogen peroxide for $2.5 \mathrm{~h}$ to quench endogenous peroxidases. After incubation in a solution for blocking non-specific antibody binding overnight at $4^{\circ} \mathrm{C}$, the tissues were incubated with a primary antibody (rabbit polyclonal anti-myosin VII, Proteus Bioscience, Ramona, CA, USA, 25-6790) at a 1:100 dilution for 4 days at $4{ }^{\circ} \mathrm{C}$ on a Nutator mixer, washed in PBS, and then incubated overnight at $4^{\circ} \mathrm{C}$ with secondary antibody (biotinylated goat anti-rabbit) at a 1:100 dilution. The specimens were rinsed again and then incubated in ABC solution (Vector Laboratories, Burlingame, CA, USA, PK-4001) overnight. Following another washing, the cochleae were incubated in $D A B$ for $3 \mathrm{~h}$, as necessary for sufficient staining intensity, followed by washing to stop the DAB reaction. Finally, the cochleae were micro-dissected under a microscope into apical, middle, and basal segments, and mounted on slides with Fluoromount-G mounting medium. Images were taken with Zeiss AxioCam MRc5 camera with Axioplan 2 imaging software (Carl Zeiss, Oberkochen, Germany), with a Zeiss microscope for hair cell counts. A sample size of five ears was used for each experimental group. Unless otherwise specified, all chemicals and reagents used were purchased from Sigma-Aldrich.

Figure 7 Protection from NIHL by a combination of SiRIP3 and ZVAD treatment is additive. (a) Treatment with ZVAD via IP injection significantly decreases threshold shifts induced by exposure to $98 \mathrm{~dB}$ SPL BBN. Data are presented as means + S.D.; $n=5-9$ for each group; ${ }^{*} P<0.05$. (b) Silencing RIP3 does not influence noise-induced thresholds. Data are presented as means + S.D.; $n=5$ for each group. (c) Additive prevention of noise-induced threshold shifts is accomplished by a combination of intratympanic delivery of siRIP3 and IP injection of ZVAD. Data are presented as means + S.D.; $n=5-9$ for each group; ${ }^{*} P<0.05$. ${ }^{\star *} P<0.001$. (d) Combination treatment also significantly reduces OHC loss. Data are presented as means + S.D.; $n=5$ for each group 


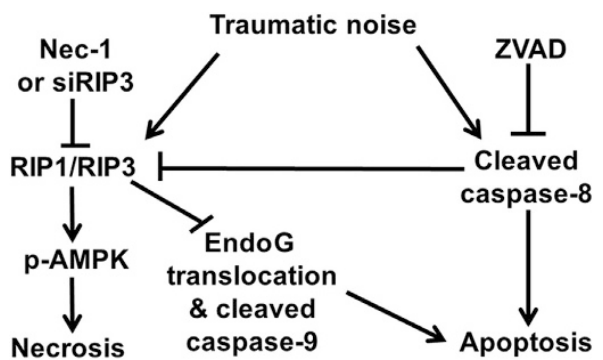

Figure 8 Summary of the molecular cell death pathways in sensory hair cells after traumatic noise exposure. Noise causes both an increase in RIP1 and RIP3 and activation of caspase-8. The activation of caspase-8 leads to apoptotic hair cell death. Inhibition of CC8 by ZVAD blocks apoptotic cell death but stimulates an increase in RIP1 and RIP3. The increase in RIP1 and RIP3 enhances P-AMPK in hair cells and results in necrotic cell death. Inhibition of RIP1 and RIP3 by Nec-1 or siRIP3 diminishes the expression of RIP1 and RIP3, but stimulates activation of caspase-9 and increases EndoG translocation to the $\mathrm{OHC}$ nuclei, resulting in apoptotic cell death. Combination treatment of ZVAD and siRIP3 provides synergetic prevention of $\mathrm{NIHL}$ and hair cell loss

Hair cell counts on cochlear epithelia from the adult mouse. Images from the apex through the base of the DAB-stained surface preparations were captured using the $\times 40$ magnification lens on the Zeiss microscope. The lengths of the cochlear epithelia were measured and recorded in millimeters. Both $\mathrm{OHCs}$ and IHCs were counted from the apex to the base along the entire length of the mouse cochlear epithelium. Percentages of hair cell loss in each $0.5 \mathrm{~mm}$ length of epithelium were plotted as a function of the cochlear length as a cytocochleogram. ${ }^{15}$

Immunocytochemistry for cochlear surface preparations. Following decalcification with 4\% EDTA, each cochlea for immunocytochemistry was dissected under a microscope by removing the softened otic capsule, stria vascularis, Reissner's membrane, and tectorial membrane. The remaining tissue, including the modiolus and cochlear sensory epithelium, was permeabilized in $3 \%$ Triton X-100 solution for $30 \mathrm{~min}$ at room temperature. The specimens were washed three times with PBS and blocked with $10 \%$ goat serum for 30 min at room temperature, followed by incubation with primary antibodies: monoclonal mouse anti-RIP1 at 1:50 (BD Biosciences, San Jose, CA, USA, 610458), polyclonal rabbit anti-RIP3 at 1:50 (Sigma-Aldrich prs2283), monoclonal rabbit anti-phospho-AMPK $\alpha$ (Thr172) at 1:50 (Cell Signaling Technology, Danvers, MA, USA, 2535), monoclonal rabbit anti-CC8 at 1:50 (Cell Signaling Technology 8592), polyclonal rabbit anti-CC9 (Cell Signaling Technology 9509), and polyclonal rabbit anti- EndoG at 1:50 (EMD Millipore Corporation, Billerica, MA, USA, AB3639) in darkness at $4{ }^{\circ} \mathrm{C}$ for $72 \mathrm{~h}$. After washing three times, the tissues were incubated with the Alexa Fluor 594- or Alexa Fluor 350-conjugated secondary antibody at a concentration of $1: 200$ at $4{ }^{\circ} \mathrm{C}$ overnight in darkness. After washing, specimens then incubated with Alexa Fluor 488 phalloidin at a concentration of $1: 100$ at room temperature for $1 \mathrm{~h}$ in darkness. For detection of apoptotic and necrotic $\mathrm{OHC}$ nuclei, surface preparations were permeabilized in 3\% Triton X-100 solution for $30 \mathrm{~min}$ at room temperature first, and then stained with PI at $1 \mu \mathrm{g} / \mathrm{ml}$ in PBS for $1 \mathrm{~h}$ at room temperature. After the final wash with PBS (10 min each), the tissue was dissected in PBS by removing the modiolus. The epithelia were divided into three segments (apex, middle, and base). Specimens were mounted on slides with Fluorogel with Tris Buffer (Electron Microscopy Sciences, Hatfield, PA, USA, 17985-10). Control incubations were routinely processed without primary antibody treatments. Immunolabeled images were taken using a Zeiss laser confocal microscope (Zeiss LSM 510).

Quantification of the immunofluorescence signals from surface preparations. Immunofluorescence of RIP1, RIP3, p-AMPK $\alpha, \mathrm{CC} 8$, and CC9 on surface preparations was quantified from original confocal images, each taken with a $\times 63$ magnification lens under identical conditions and equal parameter settings for laser gains and PMT gains, using ImageJ software (National Institutes of Health, Bethesda, MD, USA) or Zen Zeiss software. ${ }^{38}$ The cochleae from the different groups were fixed and stained simultaneously with identical solutions and processed in parallel. All of the surface preparations were counter-stained with
Alexa Fluor 488 phalloidin (green) to label hair cell structure in order to identify the comparable parts of the hair cells in confocal images. The borders of each individual $\mathrm{OHC}$ were outlined based on the phalloidin staining. The immunofluorescence of the target proteins were measured in the upper-basal region of cochlear surface preparations in $0.12-\mathrm{mm}$ segments, each containing about 60 $\mathrm{OHCs}$. The intensity of the background fluorescence was subtracted and the average fluorescence per cell was then calculated. The relative fluorescence was quantified by normalizing the ratio of average fluorescence of noise-exposed hair cells to the average fluorescence of the unexposed hair cells from the same set of the experiment using identical solution and parallel handled.

In this manuscript, all cell death signaling molecules that we investigated showed no differences in OHCs of the apical and middle turns between the ears of control mice and those collected $1 \mathrm{~h}$ after BBN noise expose. The changes occurred only in the basal turn. As the lower basal turn was affected by the exposure with considerable losses of OHCs (Figure 1), we only quantified the changes of immunofluorescence in $\mathrm{OHCs}$ in the upper basal turn where hair cells remained.

Identification of condensed or swollen $\mathrm{OHC}$ nuclei and missing OHCs. The morphological characteristics of apoptosis and necrosis have been extensively described. ${ }^{39}$ As a DNA intercalating fluorescent probe, PI was used to trace the morphological nuclear changes to identify types of $\mathrm{OHC}$ death $1 \mathrm{~h}$ after PTS noise exposure similar as reported in the literature. ${ }^{20}$ Surface preparations were first captured using a $\times 40$ magnification lens on the Zeiss microscope. The lengths of the cochlear epithelia were measured and recorded in millimeters. Next, confocal images taken with a $\times 63$ magnification lens on surface preparations of the lower basal turn (4.5-5.5 mm from apex) were used to count condensed $\mathrm{OHC}$ nuclei identified as apoptotic. Swollen nuclei that were larger than normal nuclei (diameter $\geq 7 \mu \mathrm{m}$ ) were recognized as necrotic OHCs. Missing hair cells were identified in the areas without PI staining. The percentage of condensed nuclei, swollen nuclei, and missing $\mathrm{OHCs}$ were then calculated from total abnormal OHCs.

Quantification of EndoG-positive OHC nuclei. Surface preparations were obtained from animals $1 \mathrm{~h}$ after noise exposure. EndoG-positive and EndoGnegative $\mathrm{OHC}$ nuclei were counted on confocal images (at a $\times 63$ magnification) of the lower basal turn $(4.5-5.5 \mathrm{~mm}$ from apex) in both siControl and siRIP3treated mice. From these counts, the relative number of EndoG-positive $\mathrm{OHC}$ nuclei was calculated.

Quantification of co-localization of RIP1 and RIP3 immunofluorescence. Immunofluorescence of RIP1/RIP3 co-localization on surface preparations was quantified from original confocal images, each taken with $a \times 63$ magnification lens under identical conditions and equal parameter settings for laser gains and PMT gains, using Zeiss Zen software. ${ }^{38}$ The cochleae from the different groups were fixed and stained simultaneously with identical solutions and counter-stained with phalloidin to label hair cell structure in order to identify the comparable parts of the hair cells in the confocal images. The overlap coefficients of RIP1/RIP3 fluorophores were measured per cell in the upper-basal region of cochlear surface preparations, each containing about $60 \mathrm{OHCs}$. The average overlap coefficient was then calculated per cell. Each condition was replicated in four different animals.

Extraction of total protein. Cochleae were rapidly removed and dissected in ice-cold PBS containing complete mini EDTA-free PI cocktail tablets (Roche Diagnostic GmbH, Mannheim, Germany, 11836170001) at pH 7.4. To extract total protein, tissues from the cochleae of one mouse were homogenized in ice-cold RIPA lysis buffer containing RIPA lysis buffer base ( $50 \mathrm{mM}$ Tris-HCl, $1 \%$ IGEPAL, $0.25 \%$ Na-deoxycholate, $150 \mathrm{mM} \mathrm{NaCl}, 1 \mathrm{mM}$ EDTA, $1 \mathrm{mM}$ PMSF, $1 \mathrm{mM} \mathrm{NaF}$ ) plus Phosphatase Inhibitor Cocktails II and III, and Roche protease inhibitor by using a glass/glass micro tissue grind pestle and vessel for $30 \mathrm{~s}$. After $30 \mathrm{~min}$ on ice, tissue debris were removed by centrifugation at $10000 \times g$ at $4^{\circ} \mathrm{C}$ for $10 \mathrm{~min}$ and the supernatants were retained as the total protein fractions. Protein concentrations were determined using the Bio-Rad Protein Assay dye reagent (Bio-Rad, Hercules, CA, USA) with bovine serum albumin as a protein standard. Two cochleae from the same mouse were pooled for each sample. Unless otherwise specified, all chemicals and reagents used were purchased from Sigma-Aldrich.

Western blot analysis. Protein samples $(50 \mu \mathrm{g})$ were separated by SDSPAGE. After electrophoresis, the proteins were transferred onto a nitrocellulose membrane (Pierce, Rockford, IL, USA) and blocked with $5 \%$ non-fat dry milk in 
PBS- $0.1 \%$ Tween 20 (PBS-T). The membranes were incubated with anti-RIP1 (1:1000), anti-RIP3 (1:1000), anti-p-AMPK $\alpha$ (1:1000), anti-CC8 (1:800), antiCC9 $(1: 1000)$ or anti-GAPDH (Millipore ABS16, $1: 10000)$ at $4{ }^{\circ} \mathrm{C}$ overnight, and then washed three times (10 min each) with PBS-T buffer. Membranes were incubated with an appropriate secondary antibody at a concentration of $1: 2500$ for $1 \mathrm{~h}$. Following extensive washing of the membrane, the immunoreactive bands were visualized by SuperSignal West Dura Extended Duration Substrate or Pierce ECL Western Blotting Substrate (Thermo Scientific, Waltham, MA, USA).

X-ray films of western blotting were scanned and analyzed using ImageJ software. The band densities were first normalized to background. Next, the probing protein/GAPDH ratio was calculated from the band densities run on the same gel. Finally, the difference in the ratio of the control and experimental bands was tested for statistical significance.

Mouse organotypic culture preparations. Organ culture explants were prepared as reported previously. ${ }^{34}$ Briefly, cochlear epithelia were isolated from postnatal day 3 (p3) mice. After $48 \mathrm{~h}$ of incubation to allow for recovery from dissection stress, the explants received fresh medium with individual compounds of ZVAD, Nec-1, or DMSO vehicle. After $24 \mathrm{~h}$ of incubation, explants were fixed with $4 \%$ paraformaldehyde overnight at $4{ }^{\circ} \mathrm{C}$ and then permeabilized for $30 \mathrm{~min}$ with $3 \%$ Triton X-100 in PBS at room temperature. The specimens were washed three times with PBS and incubated with Alexa Fluor 488 phalloidin $(1: 200)$ at room temperature for $30 \mathrm{~min}$. After several rinses in PBS, the specimens were mounted on a slide with Fluorogel with Tris Buffer. The phalloidin-stained stereociliary bundles and circumferential F-actin rings on the cuticular plate outlining individual hair cells were viewed on a Zeiss fluorescence microscope.

Statistical analysis. Data were analyzed using IBM SPSS Statistics Premium V21 (IBM SPSS, Armonk, NY, USA) and GraphPad software for Windows (GraphPad, La Jolla, CA, USA). Statistical methods used included oneway analysis of variance (ANOVA), two-way ANOVA, repeated-measures ANOVA, Tukey's multiple comparisons, unpaired $t$-tests, and one-sample $t$-tests. All tests were two-tailed and a $P$-value $<0.05$ was considered statistically significant.

\section{Conflict of Interest}

The authors declare no conflict of interest.

Acknowledgements. The research project described was supported by grant R01 DC009222 from the National Institute on Deafness and Other Communication Disorders, National Institutes of Health. This work was conducted in the WR Building at MUSC in renovated space supported by grant C06 RR014516. Animals were housed in MUSC CRI animal facilities supported by grant C06 RR015455 from the Extramural Research Facilities Program of the National Center for Research Resources. We thank Drs Jochen Schacht and Bradley Schulte for their valuable comments on the manuscript. We also thank statistician Dr Fu-Shing Lee for consultation of statistical analysis of the results in this study.

\section{Author contributions}

HWZ executed all experiments and analyzed results, except those from western blotting. JC conducted the western blotting and analyzed band densities. SHS designed and supervised all experiments of the manuscript, analyzed results, and wrote the manuscript.

1. Vanlangenakker N, Vanden Berghe T, Krysko DV, Festjens N, Vandenabeele P. Molecular mechanisms and pathophysiology of necrotic cell death. Curr Mol Med 2008; 8: 207-220.

2. Vandenabeele $P$, Galluzzi L, Vanden Berghe T, Kroemer G. Molecular mechanisms of necroptosis: an ordered cellular explosion. Nat Rev Mol Cell Biol 2010; 11: 700-714.

3. Hitomi J, Christofferson DE, Ng A, Yao J, Degterev A, Xavier RJ et al. Identification of a molecular signaling network that regulates a cellular necrotic cell death pathway. Cell 2008; 135: 1311-1323.

4. Trichonas G, Murakami Y, Thanos A, Morizane Y, Kayama M, Debouck CM et al. Receptor interacting protein kinases mediate retinal detachment-induced photoreceptor necrosis and compensate for inhibition of apoptosis. Proc Natl Acad Sci USA 2010; 107: 21695-21700.

5. Declercq W, Vanden Berghe T, Vandenabeele P. RIP kinases at the crossroads of cell death and survival. Cell 2009; 138: 229-232.

6. Festjens N, Vanden Berghe T, Cornelis S, Vandenabeele P. RIP1, a kinase on the crossroads of a cell's decision to live or die. Cell Death Differ 2007; 14: 400-410.
7. Lin Y, Devin A, Rodriguez Y, Liu ZG. Cleavage of the death domain kinase RIP by caspase-8 prompts TNF-induced apoptosis. Genes Dev 1999; 13: 2514-2526.

8. He S, Wang L, Miao L, Wang T, Du F, Zhao L et al. Receptor interacting protein kinase-3 determines cellular necrotic response to TNF-alpha. Cell 2009; 137: 1100-1111.

9. Cho YS, Challa S, Moquin D, Genga R, Ray TD, Guildford M et al. Phosphorylation-driven assembly of the RIP1-RIP3 complex regulates programmed necrosis and virus-induced inflammation. Cell 2009; 137: 1112-1123.

10. Vanden Berghe T, Vanlangenakker N, Parthoens E, Deckers W, Devos M, Festjens N et al. Necroptosis, necrosis and secondary necrosis converge on similar cellular disintegration features. Cell Death Differ 2010; 17: 922-930.

11. Leist M, Single B, Castoldi AF, Kuhnle S, Nicotera P. Intracellular adenosine triphosphate (ATP) concentration: a switch in the decision between apoptosis and necrosis. J Exp Med 1997; 185: 1481-1486.

12. Nagashima R, Yamaguchi T, Kuramoto N, Ogita K. Acoustic overstimulation activates 5'-AMP-activated protein kinase through a temporary decrease in ATP level in the cochlear spiral ligament prior to permanent hearing loss in mice. Neurochem Int 2011; 59: 812-820.

13. Vlajkovic SM, Housley GD, Munoz DJ, Robson SC, Sevigny J, Wang CJ et al. Noise exposure induces up-regulation of ecto-nucleoside triphosphate diphosphohydrolases 1 and 2 in rat cochlea. Neuroscience 2004; 126: 763-773.

14. Thalmann R, Miyoshi T, Thalmann I. The influence of ischemia upon the energy reserves of inner ear tissues. Laryngoscope 1972; 82: 2249-2272.

15. Chen FQ, Zheng HW, Hill K, Sha SH. Traumatic noise activates Rho-family GTPases through transient cellular energy depletion. J Neurosci 2012; 32: 12421-12430.

16. Oishi N, Schacht J. Emerging treatments for noise-induced hearing loss. Expert Opin Emerg Drugs 2011; 16: 235-245.

17. Green DR. Apoptotic pathways: ten minutes to dead. Cell 2005; 121: 671-674.

18. Joza N, Susin SA, Daugas E, Stanford WL, Cho SK, Li CY et al. Essential role of the mitochondrial apoptosis-inducing factor in programmed cell death. Nature 2001; 410 : 549-554.

19. Susin SA, Lorenzo HK, Zamzami N, Marzo I, Snow BE, Brothers GM et al. Molecular characterization of mitochondrial apoptosis-inducing factor. Nature 1999; 397: 441-446.

20. Jiang $\mathrm{H}$, Sha SH, Forge A, Schacht J. Caspase-independent pathways of hair cell death induced by kanamycin in vivo. Cell Death Differ 2006; 13: 20-30.

21. Yamashita D, Miller JM, Jiang HY, Minami SB, Schacht J. AIF and EndoG in noise-induced hearing loss. Neuroreport 2004; 15: 2719-2722.

22. Han W, Shi X, Nuttall AL. AIF and endoG translocation in noise exposure induced hair cell death. Hear Res 2006; 211: 85-95.

23. Hu BH, Henderson D, Yang WP. The impact of mitochondrial energetic dysfunction on apoptosis in outer hair cells of the cochlea following exposure to intense noise. Hear Res 2008; 236: 11-21.

24. Nicotera TM, Hu BH, Henderson D. The caspase pathway in noise-induced apoptosis of the chinchilla cochlea. J Assoc Res Otolaryngol 2003; 4: 466-477.

25. Kroemer G, Galluzzi L, Vandenabeele P, Abrams J, Alnemri ES, Baehrecke EH et al. Classification of cell death: recommendations of the Nomenclature Committee on Cell Death 2009. Cell Death Differ 2009; 16: 3-11.

26. Degterev A, Huang Z, Boyce M, Li Y, Jagtap P, Mizushima N et al. Chemical inhibitor of nonapoptotic cell death with therapeutic potential for ischemic brain injury. Nat Chem Biol 2005; 1: 112-119.

27. Newmeyer DD, Ferguson-Miller S. Mitochondria: releasing power for life and unleashing the machineries of death. Cell 2003; 112: 481-490.

28. Fujioka M, Kanzaki S, Okano HJ, Masuda M, Ogawa K, Okano H. Proinflammatory cytokines expression in noise-induced damaged cochlea. J Neurosci Res 2006; 83: 575-583.

29. Zou J, Pyykko I, Sutinen P, Toppila E. Vibration induced hearing loss in guinea pig cochlea: expression of TNF-alpha and VEGF. Hear Res 2005; 202: 13-20.

30. So H, Kim H, Lee JH, Park C, Kim Y, Kim E et al. Cisplatin cytotoxicity of auditory cells requires secretions of proinflammatory cytokines via activation of ERK and NF-kappaB. $J$ Assoc Res Otolaryngol 2007; 8: 338-355.

31. Moon SK, Park R, Lee HY, Nam GJ, Cha K, Andalibi A et al. Spiral ligament fibrocytes release chemokines in response to otitis media pathogens. Acta Otolaryngol 2006; 126: 564-569.

32. Park HJ, Kim HJ, Bae GS, Seo SW, Kim DY, Jung WS et al. Selective GSK-3beta inhibitors attenuate the cisplatin-induced cytotoxicity of auditory cells. Hear Res 2009; 257: 53-62.

33. Li J, McQuade T, Siemer AB, Napetschnig J, Moriwaki K, Hsiao YS et al. The RIP1/RIP3 necrosome forms a functional amyloid signaling complex required for programmed necrosis. Cell 2012; 150: 339-350.

34. Chen FQ, Schacht J, Sha SH. Aminoglycoside-induced histone deacetylation and hair cell death in the mouse cochlea. J Neurochem 2009; 108: 1226-1236.

35. Oishi N, Chen FQ, Zheng HW, Sha SH. Intra-tympanic delivery of short interfering RNA into the adult mouse cochlea. Hear Res 2013; 296: 36-41.

36. Mizutari K, Matsunaga T, Kamiya K, Fujinami Y, Fujii M, Ogawa K. Caspase inhibitor facilitates recovery of hearing by protecting the cochlear lateral wall from acute cochlear mitochondrial dysfunction. J Neurosci Res 2008; 86: 215-222.

37. Matsui JI, Haque A, Huss D, Messana EP, Alosi JA, Roberson DW et al. Caspase inhibitors promote vestibular hair cell survival and function after aminoglycoside treatment in vivo. J Neurosci 2003; 23: 6111-6122. 
38. Dhonukshe P, Weits DA, Cruz-Ramirez A, Deinum EE, Tindemans SH, Kakar $\mathrm{K}$ et al. A PLETHORA-auxin transcription module controls cell division plane rotation through MAP65 and CLASP. Cell 2012; 149: 383-396.

39. Galluzzi L, Vitale I, Abrams JM, Alnemri ES, Baehrecke EH, Blagosklonny MV et al. Molecular definitions of cell death subroutines: recommendations of the Nomenclature Committee on Cell Death 2012. Cell Death Differ 2012; 19: 107-120.

Cell Death and Disease is an open-access journal published by Nature Publishing Group. This work is licensed under a Creative Commons Attribution-NonCommercialShareAlike 3.0 Unported License. The images or other third party material in this article are included in the article's Creative Commons license, unless indicated otherwise in the credit line; if the material is not included under the Creative Commons license, users will need to obtain permission from the license holder to reproduce the material. To view a copy of this license, visit http://creativecommons.org/ licenses/by-nc-sa/3.0/ 